\title{
EXACT SEQUENCES FOR MIXED COPRODUCT/TENSOR-PRODUCT RING CONSTRUCTIONS
}

\author{
WARREN Dicks AND I. J. LEARY
}

\begin{abstract}
To a commutative ring $K$, and a family of $K$-algebras indexed by the vertex set of a graph, we associate a $K$-algebra obtained by a mixture of coproduct and tensor product constructions. For this, and related constructions, we give exact sequences and deduce homological properties.
\end{abstract}

\section{Introduction}

1.1. Notation. Throughout, let $K$ be a ring (associative, with 1 ). The symbols $\otimes$ and $\amalg$ with no subscript are understood to mean $\otimes_{K}$ and $\bigsqcup_{K}$, respectively.

Throughout, let $X$ be a set, and $A \subseteq X \times X$ a symmetric antireflexive relation on $X$, that is, $(x, y) \in A$ implies $(y, x) \in A$, and no $(x, x)$ lies in $A$. This corresponds to a (nonoriented) graph $X_{A}$ with vertex set $X$ : we define $X_{A}$ to have an edge connecting two elements $x$ and $y$ if and only if $(x, y) \in A$.

If $Y$ is any subset of $X$, the full subgraph $Y_{A \cap(Y \times Y)}$ of $X_{A}$ will be abbreviated $Y_{A}$.

Recall that a graph is complete if each pair of vertices is connected by an edge. Thus the complete subgraphs of $X_{A}$ correspond to the subsets $Y$ of $X$ such that any two elements of $Y$ are connected by an edge in $X_{A}$.

In this article we shall be studying a general situation described in Hypothesis 3.1, below; it is well-illustrated by the following special case. 
1.2. Construction. Suppose that $K$ is commutative, and that we are given, for each $x \in X$, a $K$-algebra $R(x)$ (associative with 1). Form the coproduct $\coprod_{x \in X} R(x)$ as $K$-algebras, and factor out the ideal generated by $\{a b-b a \mid a \in R(x), b \in R(y),(x, y) \in A\}$. Let $R\left(X_{A}\right)$ denote the resulting $K$-algebra. This construction is a mixture of the coproduct and tensor-product constructions on $K$-algebras.

For example, if $X_{A}$ is a complete graph then $R\left(X_{A}\right)=\underset{x \in X}{\otimes} R(x)$.

Our objective is to obtain homological information about $R\left(X_{A}\right)$, for example, estimates for the left global dimension. From a homological point of view the formation of tensor products of $K$-algebras is not generally well-behaved, so if we are to obtain any information at all on $R\left(X_{A}\right)$, we should have prior knowledge of, say, the $R\left(Y_{A}\right)$, for the complete subgraphs $Y_{A}$ of $X_{A}$. Thus we take certain such information as given, and try to extend it to all of $R\left(X_{A}\right)$. By constructing an exact sequence motivated in section 2 , described in section 3 , and verified in section 4 , we are able to obtain a practical upper bound for the left global dimension of $R\left(X_{A}\right)$, in terms of $X_{A}$ and the left global dimensions of rings of the form $R\left(Y_{A}\right)$, where $Y_{A}$ is a complete subgraph of $X_{A}$. In section 5 , we show how the left global dimension estimate can be refined. In section 6 we give G. M. Bergman's hitherto unpublished description of $K$-bases of $R\left(X_{A}\right)$ constructed from $K$-bases of the $R(x)$.

Consider the following group-theoretic situation.

1.3. Construction. Suppose that we are given a family of groups

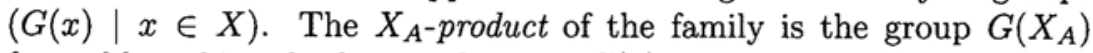
formed by taking the free product $\underset{x \in X}{*} G(x)$ as groups, and factoring out the normal subgroup generated by

$$
\left\{a b a^{-1} b^{-1} \mid a \in G(x), b \in G(y),(x, y) \in A\right\} .
$$

This construction is a mixture of the coproduct and restricted direct product constructions on groups.

For example, if $X_{A}$ is a finite complete graph then $G\left(X_{A}\right)=\underset{x \in X}{\times} G(x)$; and, for infinite complete graphs, we get the restricted direct product, generated by the $G(x)$.

For another example, if each $G(x)$ has order 2, then $G\left(X_{A}\right)$ is a rightangled Coxeter group.

Our results will give homological information on the group ring $R\left(X_{A}\right)=K\left[G\left(X_{A}\right)\right]$ in terms of the group rings $K\left[G\left(Y_{A}\right)\right]$, for certain complete subgraphs $Y_{A}$ of $X_{A}$. 
As was the case with $[\mathbf{1 0}]$, many of the results in this article are inspired by, and, in some cases, even copied from, the preprint [3] of G. M. Bergman, and must be considered joint work of the three of us. Due to other commitments, Bergman reluctantly abandoned the project, and declined all offers to be listed as a co-author on either paper. Since there now appears to be an incipient interest in Construction 1.3, cf [1], [6], [11], we have decided to go ahead and publish, with Bergman's approval.

\section{Motivating examples}

Throughout this section we consider the following.

2.1. Hypothesis. Suppose $K$ is commutative, and that we have a family of faithfully flat $K$-algebras $(R(x) \mid x \in X)$, indexed by the vertex set of the graph $X_{A}$. For any subset $Y$ of $X$, we can apply Construction 1.2 and form the $K$-algebra $R\left(Y_{A}\right)$. We set $R=R\left(X_{A}\right)$. As we shall see in Proposition 3.4, $R$ is flat as right $R\left(Y_{A}\right)$-module, for every subset $Y$ of $X$.

The following examples illustrate what we might hope to be able to say about l. gl. dim. $R$.

2.2. Example. Let $X_{A}$ be the graph

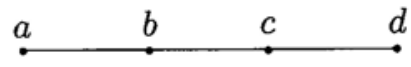

so $R=(R(a) \otimes R(b)) \coprod_{R(b)}(R(b) \otimes R(c)) \coprod_{R(c)}(R(c) \otimes R(d))$. By [9, Theorem 2] there is an exact sequence of $R$-bimodules

$$
\begin{aligned}
0 \rightarrow R \otimes_{R(b)} R \oplus R \otimes_{R(c)} R & \\
& \rightarrow R \otimes_{R(a) \otimes R(b)} R \oplus R \otimes_{R(b) \otimes R(c)} R \oplus R \otimes_{R(c) \otimes R(d)} R \\
& \rightarrow R \rightarrow 0
\end{aligned}
$$

and, as in the proof of [9, Corollary 7], since $R$ is flat as right module over $R(b), R(c), R(a) \otimes R(b), R(b) \otimes R(c)$ and $R(c) \otimes R(d)$, we have

l.gl. $\operatorname{dim} . R$

$$
\leq \max \left\{1+\text { l.gl. } \operatorname{dim} . R(x), \text { l.gl. } \operatorname{dim} . R(y) \otimes R(z) \mid x \in X_{0}, y z \in X_{1}\right\},
$$

where $X_{0}=\{b, c\}$ and $X_{1}=\{a b, b c, c d\}$. 
2.3. Example. Let $X_{A}$ be the graph

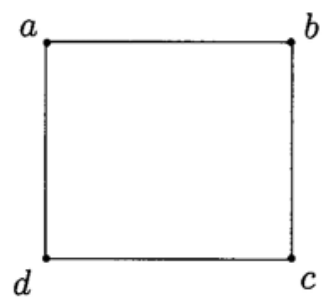

Then $R=(R(a) \amalg R(c)) \otimes(R(b) \amalg R(d))$. Consider the following subgraphs of $X_{A}$ :

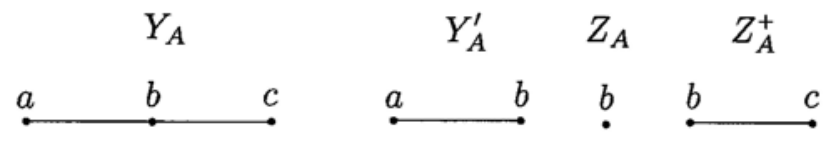

Since $R\left(Y_{A}\right)=R\left(Y_{A}^{\prime}\right) \coprod_{R\left(Z_{A}\right)} R\left(Z_{A}^{+}\right)$, by [9, Theorem 2], there is a short exact sequences of $R\left(Y_{A}\right)$-bimodules

$$
\begin{aligned}
& 0 \rightarrow R\left(Y_{A}\right) \otimes_{R\left(Z_{A}\right)} R\left(Y_{A}\right) \\
& \rightarrow R\left(Y_{A}\right) \otimes_{R\left(Y_{A}^{\prime}\right)} R\left(Y_{A}\right) \oplus R\left(Y_{A}\right) \otimes_{R\left(Z_{A}^{+}\right)} R\left(Y_{A}\right) \\
& \rightarrow R\left(Y_{A}\right) \rightarrow 0 .
\end{aligned}
$$

This is a sequence of flat right $R\left(Y_{A}\right)$-modules, and $R$ is flat as right $R\left(Y_{A}\right)$-module, so the sequence remains exact under $R \otimes_{R\left(Y_{A}\right)}-\otimes_{R\left(Y_{A}\right)} R$. This gives us a short exact sequence of $R$-bimodules which, in an obvious notation, can be abbreviated to

$$
0 \rightarrow b \rightarrow a b+b c \rightarrow a b c \rightarrow 0 .
$$

In a similar manner we can obtain other short exact sequences of $R$-bimodules which, in the same notation, are expressed as follows:

$$
\begin{gathered}
0 \rightarrow a c \rightarrow a b c+a c d \rightarrow a b c d \rightarrow 0, \\
0 \rightarrow \phi \rightarrow a+c \rightarrow a c \rightarrow 0 \\
0 \rightarrow d \rightarrow d a+c d \rightarrow a c d \rightarrow 0 .
\end{gathered}
$$


These four exact sequences can be combined to give a new exact sequence of $R$-bimodules

$$
0 \rightarrow \phi \rightarrow a+b+c+d \rightarrow a b+b c+c d+d a \rightarrow a b c d \rightarrow 0 .
$$

This sequence has the desirable property that the final term is $R$, and the other terms are induced from the rings associated with the complete subgraphs of $X_{A}$, which we have agreed to accept as our building blocks. As in the proof of [9, Corollary 7], we deduce that

l. gl. dim. $R \leq \max \{2+$ l.gl. dim. $K, 1+$ l.gl. dim. $R(x)$, 1. gl. $\left.\operatorname{dim} . R(y z) \mid x \in X_{0}, y z \in X_{1}\right\}$,

where $X_{0}=\{a, b, c, d\}$ and $X_{1}=\{a b, b c, c d, d a\}$.

To see that the term $2+$ l.gl. dim. $K$ cannot be omitted from our estimate, take the case where, for each $x \in X$,

$$
R(x)=K\left[e_{x} \mid e_{x}^{2}=e_{x}\right] \approx K \times K .
$$

Then, for all $y z \in X_{1}$,

$$
R(y z)=R(y) \otimes R(z) \approx K \times K \times K \times K .
$$

But, from [2, Examples 12.2-12.3(i)], it is known that $R(a) \amalg R(c)$ is free of rank 4 as a module over its center, a polynomial ring $K[s]$ in one indeterminate. Thus $R$ is free of rank 16 as a module over its center, a polynomial ring $K[s, t]$ in two indeterminates. It follows that

$$
\text { l. gl. } \operatorname{dim} . R=1 . \operatorname{gl} . \operatorname{dim} . K[s, t]=2+1 \text { gl. dim. } K \text {. }
$$

The next example illustrates one sort of complication that can arise as we go about constructing our exact sequences.

2.4. Example. Let $X_{A}$ be the graph

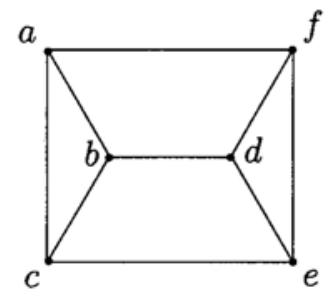


As in Example 2.3, we can construct short exact sequences of $R$-bimodules and combine them to construct an exact sequence of $R$-bimodules whose final term is $R$, and whose other terms are induced from the rings associated with the complete subgraphs of $X_{A}$. For instance, we can form the short exact sequences

$$
\begin{gathered}
0 \rightarrow a d e \rightarrow a b c d e+a d e f \rightarrow a b c d e f \rightarrow 0, \\
0 \rightarrow c d \rightarrow a b c d+c d e \rightarrow a b c d e \rightarrow 0, \\
0 \rightarrow e f \rightarrow d e f+a e f \rightarrow a d e f \rightarrow 0, \\
0 \rightarrow \phi \rightarrow a+d e \rightarrow a d e \rightarrow 0, \\
0 \rightarrow b \rightarrow a b c+b d \rightarrow a b c d \rightarrow 0, \\
0 \rightarrow e \rightarrow c e+d e \rightarrow c d e \rightarrow 0, \\
0 \rightarrow \phi \rightarrow c+d \rightarrow c d \rightarrow 0, \\
0 \rightarrow f \rightarrow a f+e f \rightarrow a e f \rightarrow 0,
\end{gathered}
$$

and combine these in stages, finally arriving at an exact sequence of $R$-bimodules

$$
\begin{aligned}
& 0 \rightarrow \phi+\phi \rightarrow a+b+c+d+e+f+d e+e f \\
& \rightarrow a f+b d+c e+d e+e f+a b c+d e f \rightarrow a b c d e f \rightarrow 0 .
\end{aligned}
$$

This exact sequence contains a redundancy, in that the component $d e+e f \rightarrow d e+e f$ is an isomorphism which can be eliminated; the process is explained in detail in section 5 . We are then left with an exact sequence of $R$-bimodules

$0 \rightarrow \phi+\phi \rightarrow a+b+c+d+e+f \rightarrow a f+b d+c e+a b c+d e f \rightarrow a b c d e f \rightarrow 0$,

and we see that here the only subgraphs that arise are the intersections of maximal complete subgraphs! The associated global dimension formula is

l.gl. dim. $R \leq \max \{2+$ l.gl. $\operatorname{dim} . K, 1+$ l.gl. $\operatorname{dim} . R(x)$, l.gl. $\left.\operatorname{dim} . R(Y) \mid x \in X_{0}, Y \in X_{1}\right\}$,

where $X_{0}=\{a, b, c, d, e, f\}$ and $X_{1}=\{a f, b d, c e, a b c, d e f\}$. 
The concluding example illustrates the sensitivity to the choice of $K$.

2.5. Example. Let $X_{A}$ be the graph

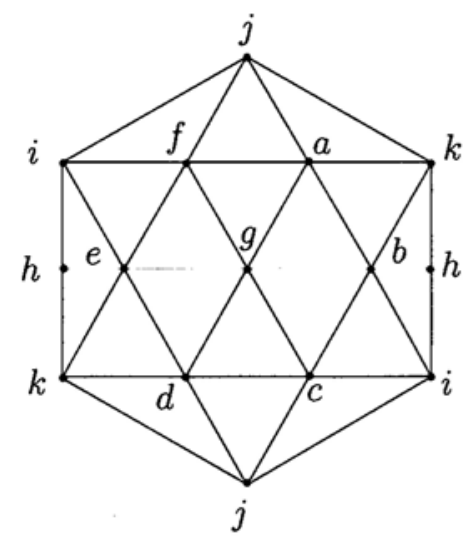

where $h, i, j, k$, and the associated edges, have been repeated, in order to achieve a planar representation. Thus $X_{A}$ arises as the 1-skeleton of a triangulation of the projective plane, which has 11 vertices, 30 edges, and 20 triangles. The triangulation is full, in the sense that every complete subgraph of the 1-skeleton, $X_{A}$, is the vertex set of a simplex. To simplify notation somewhat, let us write

$$
\begin{gathered}
X_{0}=X=\{a, b, c, d, e, f, g, h, i, j, k\}, \\
X_{1}=\{a b, a f, a g, a h, a k, a j, b c, b g, b h, b i, b k, c d, c g, c i, c j, \\
d e, d k, d j, d g, e f, e g, e h, e i, e k, f g, f i, f j, h i, h k, j k\}, \\
X_{2}=\{a b g, a b k, a f g, a f j, a j k, b c g, b c i, b h i, b h k, c d g, \\
c d j, c i j, d e g, d e k, d j k, e f g, e f i, e h i, e h k, f j i\} .
\end{gathered}
$$

By considering the circuit given by the full subgraph on $\{a, b, c, d, e, f\}$, and proceeding as in Example 2.3, we get the following exact sequence of $R$-bimodules:

$$
\begin{aligned}
0 \rightarrow \phi \rightarrow a+b+c+ & d+e+f \\
& \rightarrow a b+b c+c d+d e+e f+f a \rightarrow a b c d e f \rightarrow 0 .
\end{aligned}
$$

By considering $R(-\cup\{g\})$ in place of $R(-)$, we get a similar exact sequence:

$$
\begin{aligned}
0 \rightarrow g \rightarrow a g & +b g+c g+d g+e g+f g \\
& \rightarrow a b g+b c g+c d g+d e g+e f g+f a g \rightarrow a b c d e f g \rightarrow 0 .
\end{aligned}
$$


Continuing as before, we build up sequences:

$$
\begin{gathered}
0 \rightarrow b e \rightarrow a b c d e f g+b e h \rightarrow a b c d e f g h \rightarrow 0, \\
0 \rightarrow \phi \rightarrow b+e \rightarrow b e \rightarrow 0, \\
0 \rightarrow h \rightarrow b h+e h \rightarrow b e h \rightarrow 0, \\
0 \rightarrow f e h b c \rightarrow a b c d e f g h+f e h b c i \rightarrow a b c d e f g h i \rightarrow 0, \\
0 \rightarrow e+h+b \rightarrow f e+e h+h b+b c \rightarrow f e h b c \rightarrow 0, \\
0 \rightarrow e i+h i+b i \rightarrow f e i+e h i+h b i+b c i \rightarrow f e h b c i \rightarrow 0, \\
0 \rightarrow d c i f a \rightarrow a b c d e f g h i+d c i f a j \rightarrow a b c d e f g h i j \rightarrow 0, \\
0 \rightarrow c+i+f \rightarrow d c+c i+i f+f a \rightarrow d c i f a \rightarrow 0, \\
0 \rightarrow c j+i j+f j \rightarrow d c j+c i j+i f j+f a j \rightarrow d c i f a j \rightarrow 0, \\
0 \rightarrow a b h e d j \rightarrow a b c d e f g h i j+a b h e d j k \rightarrow a b c d e f g h i j k \rightarrow 0, \\
0 \rightarrow \phi \rightarrow b+h+e+d+j+a \\
\rightarrow a b+b h+h e+e d+d j+j a \rightarrow a b h e d j \rightarrow 0, \\
0 \rightarrow k \rightarrow b k+h k+e k+d k+j k+a k \\
\rightarrow a b k+b h k+h e k+e d k+d j k+j a k \rightarrow a b h e d j k \rightarrow 0 .
\end{gathered}
$$

These then combine to give an exact sequence

$$
\begin{aligned}
& 0 \rightarrow \phi \rightarrow \phi+b+e++\sum_{x \in X_{0}} x \\
& \rightarrow b+e+h+b h+e h+\sum_{x y \in X_{1}} x y \\
& \quad \rightarrow b h+e h+\sum_{x y z \in X_{2}} x y z \rightarrow a b c d e f g h i j k \rightarrow 0 .
\end{aligned}
$$

Here we have three components $b+e+h \rightarrow b+e+h, b h+e h \rightarrow b h+e h$, and $\phi \rightarrow \phi$, which we might hope to eliminate. It turns out that the first two are isomorphisms, and can be eliminated, but the third is given by multiplication by 2 , which is an isomorphism if and only if 2 is invertible in $R$, which is equivalent to 2 being invertible in $K$, by the faithful flatness assumptions.

Thus, in general, we have an exact sequence

$$
0 \rightarrow \phi \rightarrow \phi+\sum_{x \in X_{0}} x \rightarrow \sum_{x y \in X_{1}} x y \rightarrow \sum_{x y z \in X_{2}} x y z \rightarrow \text { abcdefghijk } \rightarrow 0 .
$$

There is an underlying exact sequence obtained by taking all the $R(x)$ to be $\mathbb{Z}$, and it is the augmented chain complex of a contractible threedimensional $\mathrm{CW}$-complex with 20 vertices, 30 edges, 12 polygonal faces, 
and 1 three-cell. The CW-complex arises by taking the dual of the given tessellation of the projective plane, adding on a polygonal disk to kill the fundamental group, which here coincides with the homology group in dimension 1, and adding on a three-cell to kill the resulting homology in dimension 2. It was not obvious at the outset that the relatively unsophisticated algebraic process of forming exact sequences, and then refining them, had to yield such a relatively sophisticated topological object, although it was encouraged in that direction by the original choice of a full triangulation of the projective plane.

One consequence is that

l. gl. $\operatorname{dim} . R \leq \max \{3+$ l.gl. dim. $K$,

$$
\begin{aligned}
& 2+\text { l.gl. dim. } R(x), 1+\text { l.gl. dim. } R(x y), \\
& \left.\quad \text { l.gl. } \operatorname{dim} . R(x y z) \mid x \in X_{0}, x y \in X_{1}, x y z \in X_{2}\right\} .
\end{aligned}
$$

If $\frac{1}{2} \in K$, then we also have an exact sequence

$$
0 \rightarrow \sum_{x \in X_{0}} x \rightarrow \sum_{x y \in X_{1}} x y \rightarrow \sum_{x y z \in X_{2}} x y z \rightarrow \text { abcdefghijk } \rightarrow 0,
$$

so that here the term $3+\mathrm{l}$.gl. dim. $K$ can be omitted from our previous estimate.

To see that the term $3+$ l.gl. dim. $K$ cannot be omitted in general, take $K$ to be a field, and, for each $x \in X$, take

$$
R(x)=K\left[e_{x} \mid e_{x}^{2}=e_{x}\right] \approx K \times K .
$$

Let $I=\sum_{x \in X} R e_{x}$, so $I$ is a two-sided ideal of $R$ such that $R=K \oplus I$. Let $K_{\varepsilon}$ denote the $R$-bimodule $R / I$. Then it is not difficult to use the resolution to show that $\operatorname{Ext}_{R}^{3}\left(K_{\varepsilon}, R\right) \approx K_{\varepsilon} / 2 K_{\varepsilon}$, as $R$-bimodules. Hence if $K$ has characteristic 2 then l.gl. $\operatorname{dim} . R \geq 3$, while if $K$ has characteristic different from 2 then l.gl. dim. $R \leq 2$. In fact equality holds in both cases, the reverse inequalities coming from our estimate, and from a surjective map $\operatorname{Ext}_{R}^{2}\left(K_{\varepsilon}, R\right) \rightarrow K_{\varepsilon}$, respectively.

This example is closely related to one of the right-angled Coxeter groups which have virtual cohomological dimension 2 over $\mathbb{Q}$, and 3 over $\mathbb{Z}$, described by $M$. Bestvina [5, Remark (3)].

What general pattern emerges from these examples? It is clear that for any graph $X_{A}$ (even infinite) this procedure, of combining exact sequences and eliminating redundancies, will always give an exact sequence that gives information about $R\left(X_{A}\right)$ in terms of the rings associated with 
the complete subgraphs. What is not immediately clear is to what extent the sequence is unique (depending as it does on a prechosen vertex-byvertex build up of $X_{A}$ ), which complete subgraphs will occur, and what general conclusions can be drawn about $R\left(X_{A}\right)$. In the next section we shall see more clearly what is happening.

\section{Description of the objective}

The general situation which we shall be considering is the following.

3.1. Hypothesis. (i) Let $X_{A}$ be a graph.

(ii) Let $(R(Y) \mid Y \subseteq X)$ be a directed system of rings indexed by the subsets of $X$.

We write $K=R(\phi), R=R(X)$, and, for each $x \in X, R(x)=R(\{x\})$.

(iii) Suppose that, for each $Y \subseteq X, R(Y)$ is generated by the union of the images of the $R(y), y \in Y$.

(iv) Suppose that, for each non-empty finite subset $Y$ of $X$, and each element $w$ of $Y$, for $Z=\operatorname{link}_{Y_{A}}(w)$, the induced ring homomorphism, from $R(Y-\{w\}) \underset{R(Z)}{\coprod} R(Z \cup\{w\})$ to $R(Y)$, is an isomorphism.

$(v)$ Suppose that, for all $w \in X$, and all finite $Z \subseteq \operatorname{link}_{X_{A}}(w)$, the ring $R(Z \cup\{w\})$ is faithfully flat as right $R(Z)$-module.

(vi) Fix a ring $k$ which is a subring of $\mathbb{Q}$ or a prime field, and such that $k$ maps to $K$.

We offer the following commentary to try to make clear what we mean here.

3.2. Notes. (i) We shall see in Proposition 3.4 that the directed system of rings $(R(Y) \mid Y \subseteq X)$ can be viewed as a directed system of subrings of $R$, as all the maps are inclusions.

(ii) By link $Y_{Y_{A}}(w)$ we mean the set of vertices of $Y_{A}$ joined by an edge to $w$; in particular, $w \notin \operatorname{link}_{Y_{A}}(w)$.

(iii) In $3.1(v)$, that $R(Z \cup\{w\})$ is faithfully flat as right $R(Z)$-module, means that the given map from $R(Z)$ to $R(Z \cup\{w\})$ is injective, and the cokernel is flat as right $R(Z)$-module. Notice that $3.1(v)$ is implied by the pair of conditions:

(a) for all $w \in X, R(w)$ is faithfully flat as right $K$-module;

(b) for all $w \in X$, and all $Z \subseteq \operatorname{link}_{X_{A}}(w)$, the induced right $R(Z)$-module map from $R(w) \otimes R(Z)$ to $R(Z \cup\{w\})$ is an isomorphism.

In this event, $R$ can be built up from the $R(x)$ using ring coproducts, tensor-products over $K$ with unspecified ring structure, and directed unions. 
(iv) In section 5 we shall describe the rôle we want $k$ to play in simplifying an exact sequence. We could allow $k$ to be any (commutative) principal ideal domain which maps to the intersection of $K$ with the centre of $R$. The most natural choice for $k$ is $\mathbb{Z}$, but Example 2.5 indicates the importance of allowing more general $k$.

3.3. Examples. (i) Let $K$ be a commutative ring, and

$$
(R(x) \mid x \in X)
$$

a family of faithfully flat $K$-algebras. For any subset $Y$ of $X$, we can form the $K$-algebra $R(Y)=R\left(Y_{A}\right)$, as in Construction 1.2. This gives a directed system, and one readily verifies that Hypothesis 3.1 holds. Here, the conditions $(a),(b)$ of $3.2(i i i)$ hold, since both terms in $(b)$ can be identified with the $K$-algebra $R(w) \otimes R(Z)$.

Even in this case it is not immediately obvious that all the maps involved are injective.

(ii) Consider a family of group rings $\left(R(Y)=K\left[G\left(Y_{A}\right)\right] \mid Y \subseteq X\right)$, with groups $G\left(Y_{A}\right)$ arising as in Construction 1.3. Again it is readily verified that Hypothesis 3.1 holds.

3.4. Proposition. If Hypothesis 3.1 holds, then $R(X)$ is faithfully flat as right $R(Y)$-module, for every subset $Y$ of $X$.

Proof: By 3.1 (iii), it suffices to show that $R(X)$ is faithfully flat as right $R\left(Y^{\prime}\right)$-module, for every finite subset $Y^{\prime}$ of $Y$. Thus we may assume that $Y$ is finite. Also by $3.1(i i i)$, it suffices to show that for any finite subset $X^{\prime}$ of $X$ containing $Y, R\left(X^{\prime}\right)$ is faithfully flat as right $R(Y)$-module. Thus we may asssume that $X$ is finite, and proceed by induction on $|X|$. If $X$ is empty, the statement of the proposition holds, so we may suppose that $X$ is nonempty, and that the statement holds whenever $X$ is replaced by any proper subset of $X$. Let $w \in X$, and let $Z=\operatorname{link}_{X_{A}}(w)$. By 3.1(iv), $R(X)=R(X-\{w\}) \underset{R(Z)}{\amalg} R(Z \cup\{w\})$. By the induction hypothesis, $R(X-\{w\})$ is faithfully flat as right $R(Z)$-module. By $3.1(v), R(Z \cup\{w\})$ is faithfully flat as right $R(Z)$-module. Now by a theorem of Cohn [7, Theorem 4.4], the coproduct, $R(X)$, is faithfully flat as right $R(X-\{w\})$-module. By transitivity of faithful flatness, $R(X)$ is faithfully flat as right $R(Y)$-module for any subset $Y$ of $X$ not containing $w$. Since $w$ was arbitrary, the result follows.

In section 6 we give a result of Bergman's, which shows how "faithfully flat" can be replaced with "free on a basis containing 1", and gives an explicit description of the resulting basis. 
3.5. Definitions. By an augmented simplicial complex $\mathcal{C}_{*}$ we mean a non-empty set of finite subsets of some (possibly empty) set $S$, such that every subset of an element of $\mathcal{C}_{*}$ is again in $\mathcal{C}_{*}$, that is, $\mathcal{C}_{*}$ is closed under taking subsets.

Let us write $\mathbb{N}$ for the set of non-negative integers, and $\mathbb{N}-1$ for the set of integers greater than, or equal to, -1 .

For each $n \in \mathbb{N}-1$, we let $\mathcal{C}_{n}$ denote the set consisting of those sets in $\mathcal{C}_{*}$ which have exactly $n+1$ elements. The elements of $\mathcal{C}_{n}$ are called the $n$-simplices, and are said to have dimension $n$. The 0 -simplices are called the vertices of $\mathcal{C}_{*}$. The dimension of $\mathcal{C}_{*}$, denoted $\operatorname{dim} \mathcal{C}_{*}$, is the supremum of the dimensions of the simplices; this is either $\infty$, or an element of $\mathbb{N}-1$.

If we delete the simplex of dimension -1 from $\mathcal{C}_{*}$, we get a simplicial complex, denoted $\mathcal{C}_{*_{+}}$.

In practice, we usually think of $\mathcal{C}_{*}$ as a subset of the free exterior monoid on $S$, and, in particular, blur the distinction between a 0 -simplex and an element of $S$. It is customary to identify, loosely, $S=\mathcal{C}_{0}$.

For any poset $\left(\mathcal{P}_{0}, \prec\right)$, there is an associated augmented simplicial complex, $\mathcal{P}_{*}$, which is the augmented simplicial complex whose vertex set is $\mathcal{P}_{0}$, and whose simplices are the finite subsets $\left\{p_{0}, \ldots, p_{n}\right\}$ of $\mathcal{P}_{0}$, such that $n \in \mathbb{N}-1$, and $p_{0} \prec \cdots \prec p_{n}$.

3.6. Definitions. We write $\mathcal{C}_{0} X_{A}$ to denote the set of vertex sets of complete subgraphs of $X_{A}$, and $\mathcal{F}_{0} X_{A}$ to denote the set of vertex sets of finite complete subgraphs of $X_{A}$, and $\mathcal{M}_{0} X_{A}$ to denote the set of vertex sets of maximal complete subgraphs of $X_{A}$, and $\mathcal{I}_{0} X_{A}$ to denote the set of vertex sets of those subgraphs of $X_{A}$ which are intersections of finitely many maximal complete subgraphs of $X_{A}$.

Thus $\mathcal{M}_{0} X_{A} \subseteq \mathcal{I}_{0} X_{A} \subseteq \mathcal{C}_{0} X_{A} \supseteq \mathcal{F}_{0} X_{A}$, and, if $X_{A}$ is finite, then $\mathcal{F}_{0} X_{A}=\mathcal{C}_{0} X_{A}$.

Here $\mathcal{C}_{0} X_{A}$ is a poset, with the partial order being given by inclusion, and $\mathcal{I}_{0} X_{A}, \mathcal{F}_{0} X_{A}$ are sub-posets. Let $\mathcal{C}_{*} X_{A}, \mathcal{I}_{*} X_{A}$, and $\mathcal{F}_{*} X_{A}$ denote the augmented simplicial complexes associated with the posets $\mathcal{C}_{0} X_{A}$, $\mathcal{I}_{0} X_{A}$, and $\mathcal{F}_{0} X_{A}$, respectively. Let $\mathcal{M}_{*} X_{A}$ be the set of all finite subsets of $\mathcal{M}_{0} X_{A}$; if we want to view this as the augmented simplicial complex associated with a poset, we have to choose a total ordering of $\mathcal{M}_{0} X_{A}$.

We say that $X_{A}$ is a finite-dimensional graph if $\mathcal{I}_{*} X_{A}$ is finite-dimensional; that is, there is a finite bound $d=\operatorname{dim} \mathcal{I}_{*} X_{A}$ on the set of integers $n$ such that there exists a chain $X_{0} \subset \cdots \subset X_{n}$ in $\mathcal{I}_{0} X_{A}$.

Let $Y \in \mathcal{I}_{0} X_{A}$.

Let $\mathcal{I}_{0}\left(Y, X_{A}\right)$ denote the sub-poset $\left\{Z \in \mathcal{I}_{0} X_{A} \mid Z \supset Y\right\}$ of $\mathcal{I}_{0} X_{A}$, and let $\mathcal{I}_{*}\left(Y, X_{A}\right)$ denote the associated augmented simplicial complex; 
thus

$$
\mathcal{I}_{*}\left(Y, X_{A}\right)=\left\{\left\{X_{0}, \ldots, X_{n}\right\} \subseteq \mathcal{I}_{0} X_{A} \mid n \in \mathbb{N}-1, Y \subset X_{0} \subset \cdots \subset X_{n}\right\} .
$$

The $\mathcal{I}_{*}\left(Y, X_{A}\right)$ contain much information of interest to us.

The depth of $Y$ with respect to $X_{A}$, denoted $\mathrm{d}\left(Y, X_{A}\right)$, is defined to be $\operatorname{dim} \mathcal{I}_{*}\left(Y, X_{A}\right)+1$.

Let $k$ be a principal ideal domain. The homology group of $\mathcal{I}_{*}\left(Y, X_{A}\right)$ with coefficients in $k$ is denoted $H_{*}\left(\mathcal{I}_{*}\left(Y, X_{A}\right), k\right)$. The homological depth over $k$ of $Y$ with respect to $X_{A}$, denoted $\operatorname{hd}_{k}\left(Y, X_{A}\right)$, is defined to be the infimum of the integers $n$ such that $H_{n-1}\left(\mathcal{I}_{*}\left(Y, X_{A}\right), k\right)$ is a free (possibly trivial) $k$-module, and, for all $m \geq n, H_{m}\left(\mathcal{I}_{*}\left(Y, X_{A}\right), k\right)=0$. Since $k$ is a principal ideal domain, it is not difficult to see that $\operatorname{hd}_{k}\left(Y, X_{A}\right) \leq \mathrm{d}\left(Y, X_{A}\right)$. For example, $\operatorname{hd}_{k}\left(Y, X_{A}\right)=-\infty$ if $\mathcal{I}_{*}\left(Y, X_{A}\right)$ is contractible; $\operatorname{hd}_{k}\left(Y, X_{A}\right)=0$ if and only if $\mathcal{I}_{*}\left(Y, X_{A}\right)=\phi$, that is, $Y \in \mathcal{M}_{0} X_{A}$.

In Theorem 4.6 we shall prove that, if Hypothesis 3.1 holds, then there is an exact sequence of $R$-bimodules

$$
\begin{aligned}
\cdots \rightarrow \underset{\left\{X_{0}, \ldots, X_{n}\right\} \in \mathcal{I}_{n} X_{A}}{\oplus} R \otimes_{R\left(X_{0}, \ldots, X_{n}\right)} R \stackrel{\partial_{n}}{\longrightarrow} \ldots \\
\quad \ldots \rightarrow \underset{X_{0} \in \mathcal{I}_{0} X_{A}}{\oplus} R \otimes_{R\left(X_{0}\right)} R \rightarrow R \rightarrow 0,
\end{aligned}
$$

with $\partial_{n}$ given by $\left(a \otimes_{R\left(X_{0}, \ldots, X_{n}\right)} b\right) \partial_{n}=\sum_{i=0}^{n}(-1)^{i} a \otimes_{R\left(X_{0}, \ldots \hat{X}_{i} \ldots, X_{n}\right)} b$, where $\hat{X}_{i}$ means that the term $X_{i}$ is omitted, and $R\left(X_{0}, \ldots, X_{n}\right)$ denotes $R\left(X_{0} \cap \cdots \cap X_{n}\right)$, which is just $R\left(X_{0}\right)$, since $X_{0} \subset \cdots \subset X_{n}$, by convention.

In Corollary 4.8 we deduce that, when Hypothesis 3.1 holds,

$$
\text { l. gl. } \operatorname{dim} . R \leq \sup \left\{\mathrm{d}\left(Y, X_{A}\right)+\text { l.gl. } \operatorname{dim} . R(Y) \mid Y \in \mathcal{I}_{0} X_{A}\right\} \text {. }
$$

As in Examples 2.4 and 2.5, there can be redundancies in the resolution (1). In section 5, assuming $X_{A}$ finite-dimensional, we show how to use the fact that $k$ is a principal ideal domain to eliminate redundancies to obtain, in Theorem 5.6, an exact sequence of $R$-bimodules with canonical terms, but not canonical maps. Corollary 5.7 shows that, if Hypothesis 3.1 holds and $X_{A}$ is finite-dimensional, then

$$
\text { l. gl. dim. } R \leq \sup \left\{\operatorname{hd}_{k}\left(Y, X_{A}\right)+\text { l.gl. dim. } R(Y) \mid Y \in \mathcal{I}_{0} X_{A}\right\} .
$$

As a general bound, (2) is quite satisfactory. It gives precisely the estimates obtained in Examples 2.2, 2.3 and 2.5. But, for Example 2.4 
(resp. Example 2.5 with $\frac{1}{2} \in K$ ), (2) adds in an unnecessary term of $2+$ l. gl. dim. $K$ (resp. $3+$ l. gl. dim. $K$ ). This can be remedied by using (3) with $k=\mathbb{Z}$ (resp. $k=\mathbb{Z}\left[\frac{1}{2}\right]$ ), but the bound in (3) has the disadvantage of being difficult to compute, and it is not valid for arbitrary (infinitedimensional) graphs, cf. Example 5.8.

If we have a family $(G(x) \mid x \in X)$ of groups, and we form $G=G\left(X_{A}\right)$ as in Construction 1.3, then, for the cohomological dimension of $G$ with respect to $K$, we get the estimate

$$
\operatorname{cd}_{K} G\left(X_{A}\right) \leq \sup \left\{\mathrm{d}\left(Y, X_{A}\right)+\operatorname{cd}_{K} G\left(Y_{A}\right) \mid Y \in \mathcal{I}_{0} X_{A}\right\},
$$

in terms of the groups arising from finite intersections of maximal complete subgraphs of $X_{A}$. If $X_{A}$ is finite-dimensional, then

$$
\operatorname{cd}_{K} G\left(X_{A}\right) \leq \sup \left\{\operatorname{hd}_{k}\left(Y, X_{A}\right)+\operatorname{cd}_{K} G\left(Y_{A}\right) \mid Y \in \mathcal{I}_{0} X_{A}\right\} .
$$

\section{Various resolutions}

Throughout this section, suppose that Hypothesis 3.1 holds.

4.1. Notation. Let $Y$ be a subset of $X$.

Let $R\left(\mathcal{C}_{*} Y\right)$ denote the $R$-bimodule presented on a generating set consisting of all expressions

$$
\left\langle X_{0}, \ldots, X_{n}\right\rangle \text {, such that } n \in \mathbb{N}-1 \text {, and } X_{0} \subseteq \cdots \subseteq X_{n} \text { in } \mathcal{C}_{0} Y_{A} \text {, }
$$

and relations saying that each $\left\langle X_{0}, \ldots, X_{n}\right\rangle$ is centralized by

$$
R\left(X_{0}, \ldots, X_{n}\right)=\left\{\begin{array}{l}
R\left(X_{0}\right) \text { if } n \in \mathbb{N} \\
R(Y) \text { if } n=-1
\end{array}\right.
$$

and vanishes if two of the $X_{i}$ are equal. Thus

$$
R\left(\mathcal{C}_{*} Y\right) \approx \bigoplus\left(R \otimes_{R\left(X_{0}, \ldots, X_{n}\right)} R \mid n \in \mathbb{N}-1, X_{0} \subset \cdots \subset X_{n} \text { in } \mathcal{C}_{0} Y_{A}\right)
$$

We grade $R\left(\mathcal{C}_{*} Y\right)$ by declaring that each nonzero $\left\langle X_{0}, \ldots, X_{n}\right\rangle$ is homogeneous of degree $n$.

There is an $R$-bimodule differential $\partial_{*}: R\left(\mathcal{C}_{*} Y\right) \rightarrow R\left(\mathcal{C}_{*} Y\right)$ defined on generators by $\left\langle X_{0}, \ldots, X_{n}\right\rangle \partial_{n}=\sum_{i=0}^{n}(-1)^{i}\left\langle X_{0}, \ldots \hat{X}_{i} \ldots, X_{n}\right\rangle$, where the empty sum is 0 . It is readily verified that $\partial_{*}$ is well-defined. Thus $R\left(\mathcal{C}_{*} Y\right)$ is a differential graded $R$-bimodule. Its homology group is a graded $R$-bimodule; it will be denoted $H_{*}\left(R\left(\mathcal{C}_{*} Y\right)\right)$. 


\subsection{Notation. Let $Y$ be a subset of $X$.}

We write $R\left(\mathcal{I}_{*} Y\right)$ (resp. $R\left(\mathcal{F}_{*} Y\right)$ ) to denote the $R$-sub-bimodule of $R\left(\mathcal{C}_{*} Y\right)$ generated by those $\left\langle X_{0}, \ldots, X_{n}\right\rangle, n \in \mathbb{N}-1$, for which the $X_{i}$ lie in $\mathcal{I}_{0} Y_{A}$ (resp. $\left.\mathcal{F}_{0} Y_{A}\right)$; thus $R\left(\mathcal{I}_{*} X\right)$ looks like (1). The homology $R$-bimodule will be denoted $H_{*}\left(R\left(\mathcal{I}_{*} Y\right)\right)$ (resp. $H_{*}\left(R\left(\mathcal{F}_{*} Y\right)\right)$ ).

4.3. Notation. Let $Y$ be a subset of $X$.

We write $R\left(\mathcal{M}_{*} Y\right)$ to denote the $R$-bimodule presented on a generating set consisting of all expressions

$$
\left\langle Y_{0}, \ldots, Y_{n}\right\rangle \text {, where } n \in \mathbb{N}-1, Y_{0}, \ldots, Y_{n} \in \mathcal{M}_{0} Y_{A},
$$

and relations saying that each $\left\langle Y_{0}, \ldots, Y_{n}\right\rangle$ is centralized by

$$
R\left(Y_{0}, \ldots, Y_{n}\right)= \begin{cases}R\left(Y_{0} \cap \cdots \cap Y_{n}\right) & \text { if } n \in \mathbb{N} \\ R(Y) & \text { if } n=-1,\end{cases}
$$

and vanishes if two of the $Y_{i}$ are equal, and, for any permutation $\pi$ of $\{0, \ldots, n\}$, we have $\left\langle Y_{\pi(0)}, \ldots, Y_{\pi(n)}\right\rangle=\operatorname{sign} \pi\left\langle Y_{0}, \ldots, Y_{n}\right\rangle$. It is not difficult to see that, for any total order $\prec$ on $\mathcal{M}_{0} Y_{A}$, we have

$$
R\left(\mathcal{M}_{*} Y\right) \approx \bigoplus\left(R \otimes_{R\left(Y_{0}, \ldots, Y_{n}\right)} R \mid n \in \mathbb{N}-1, Y_{0} \prec \cdots \prec Y_{n} \text { in } \mathcal{M}_{0} X_{A}\right) .
$$

We grade $R\left(\mathcal{M}_{*} Y\right)$ by declaring that each nonzero $\left\langle Y_{0}, \ldots, Y_{n}\right\rangle$ is homogeneous of degree $n$.

There is an $R$-bimodule differential $\partial_{*}: R\left(\mathcal{M}_{*} Y\right) \rightarrow R\left(\mathcal{M}_{*} Y\right)$ defined on generators by $\left\langle Y_{0}, \ldots, Y_{n}\right\rangle \partial_{n}=\sum_{i=0}^{n}(-1)^{i}\left\langle Y_{0}, \ldots \hat{Y}_{i} \ldots, Y_{n}\right\rangle$, where the empty sum is 0 . It is readily verified that $\partial_{*}$ is well-defined. Thus $R\left(\mathcal{M}_{*} Y\right)$ is a differential graded $R$-bimodule. The homology $R$-bimodule will be denoted $H_{*}\left(R\left(\mathcal{M}_{*} Y\right)\right)$.

We will show that all these augmented chain complexes are acyclic, that is, their homology groups are all 0 . We start with the simplest case.

4.4. Theorem. If Hypothesis 3.1 holds, then, for any subset $Y$ of $X$, $R\left(\mathcal{F}_{*} Y\right)$ is acyclic.

Proof: We consider first the case where $Y$ is finite, and argue by induction on $|Y|$.

If $|Y|=0$ then $R\left(\mathcal{F}_{*} Y\right)$ is generated by $\langle Y\rangle$ and \langle\rangle , which centralize $R(Y)=R\left(\right.$ ), and we see $H_{*}\left(R\left(\mathcal{F}_{*} Y\right)\right)=0$.

Now assume that $|Y| \geq 1$, and that $H_{*}\left(R\left(\mathcal{F}_{*} Y^{\prime}\right)\right)=0$ for all proper subsets $Y^{\prime}$ of $Y$. 
If $Y_{A}$ is complete then there is a well-defined, degree $+1, R$-bimodule endomorphism $s_{*}$ of $R\left(\mathcal{F}_{*} Y\right)$ which is given on generators by

$$
\left\langle X_{0}, \ldots, X_{m}\right\rangle s_{m}=(-1)^{m+1}\left\langle X_{0}, \ldots, X_{m}, Y\right\rangle .
$$

It is readily verified that $s_{*}$ is well-defined, and that $s_{*} \partial_{*}+\partial_{*} s_{*}$ acts as the identity on $R\left(\mathcal{F}_{*} Y\right)$. Thus

$$
\operatorname{Ker} \partial_{*}=\left(\operatorname{Ker} \partial_{*}\right)\left(s_{*} \partial_{*}+\partial_{*} s_{*}\right)=\left(\operatorname{Ker} \partial_{*}\right)\left(s_{*} \partial_{*}\right) \subseteq \operatorname{Im} \partial_{*},
$$

so $H_{*}\left(R\left(\mathcal{F}_{*} Y\right)\right)=0$, in this case.

Thus we may assume that $Y_{A}$ is not complete, so there exist two vertices $v, w$ of $Y_{A}$ which are not joined by an edge. Let $Z=\operatorname{link}_{Y_{A}}(w)$, let $W=Z \cup\{w\}$, and let $U=Y-\{w\}$.

Since $Z \subset W \subseteq Y-\{v\}$, we see that $Z, W$, and $U$ are proper subsets of $Y$. So, by the induction hypothesis, $H_{*}\left(R\left(\mathcal{F}_{*} Z\right)\right), H_{*}\left(R\left(\mathcal{F}_{*} W\right)\right)$, and $H_{*}\left(R\left(\mathcal{F}_{*} U\right)\right)$ are all 0 .

Clearly $U_{A} \cap W_{A}=Z_{A}$ and $U_{A} \cup W_{A}=Y_{A}$.

Notice that we have $\mathcal{F}_{*} U_{A} \cap \mathcal{F}_{*} W_{A}=\mathcal{F}_{*} Z_{A}$ in $\mathcal{F}_{*} Y_{A}$.

We claim that $\mathcal{F}_{*} U_{A} \cup \mathcal{F}_{*} W_{A}=\mathcal{F}_{*} Y_{A}$. Consider any element $z \in \mathcal{F}_{*} Y_{A}$ so $z=\left\{X_{0}, \ldots, X_{n}\right\} \subseteq \mathcal{F}_{0} Y_{A}$, where $X_{0} \subset \cdots \subset X_{n}$. If $X_{n}$ does not contain $w$, then $X_{n} \subseteq U$, so $z \in \mathcal{F}_{*} U_{A}$. On the other hand, if $X_{n}$ does contain $w$, then, since $X_{n}$ is complete, $X_{n} \subseteq \operatorname{link}_{Y_{A}}(w) \cup\{w\}=W$. Thus $z \in \mathcal{F}_{*} W_{A}$. This proves $\mathcal{F}_{*} Y_{A} \subseteq \mathcal{F}_{*} U_{A} \cup \mathcal{F}_{*} W_{A}$, and the reverse inclusion is obvious.

It now follows easily that the natural sequence of differential graded $R$-bimodules

$$
0 \rightarrow R\left(\mathcal{F}_{*} Z\right) \stackrel{(++)}{\longrightarrow} R\left(\mathcal{F}_{*} W\right) \oplus R\left(\mathcal{F}_{*} U\right) \stackrel{\left({ }_{+}^{+}\right)}{\longrightarrow} R\left(\mathcal{F}_{*} Y\right) \rightarrow 0
$$

is exact in all non-negative degrees. The degree -1 part of (4) has the form

(5) $\quad 0 \rightarrow R \otimes_{R(Z)} R \stackrel{(++)}{\longrightarrow} R \otimes_{R(U)} R \oplus R \otimes_{R(W)} R \stackrel{\stackrel{(-)}{\longrightarrow}}{\longrightarrow} R \otimes_{R(Y)} R \rightarrow 0$, and we now show this is exact. By $3.1(i v), R(Y)=R(U) \coprod_{R(Z)} R(W)$, so, by [9, Theorem 2], there is a short exact sequence of $R(Y)$-bimodules

$$
\begin{aligned}
0 \rightarrow R(Y) \otimes_{R(Z)} R(Y) & \\
\stackrel{(++)}{\longrightarrow} R(Y) \otimes_{R(U)} R(Y) \oplus R(Y) \otimes_{R(W)} R(Y) & \stackrel{(-)}{\longrightarrow} R(Y) \rightarrow 0 .
\end{aligned}
$$


By Proposition 3.4, this is a resolution of 0 by (faithfully) flat right $R(Y)$-modules, so remains exact under $-\otimes_{R(Y)} R$, since the resulting homology is $\operatorname{Tor}_{*}^{R(Y)}(0, R)=0$. Again by Proposition 3.4, $R$ is flat as right $R(Y)$-module, so the resulting sequence remains exact under $R \otimes_{R(Y)}{ }^{-}$, giving the exact sequence (5). Thus (4) is a short exact sequence of differential graded $R$-bimodules. Applying the homology functor, we get a natural exact triangle

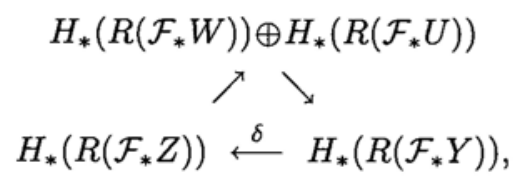

where $\delta$ has degree -1 . Since we have verified that the groups at two vertices of the triangle are zero, it follows immediately that the third group, $H_{*}\left(R\left(\mathcal{F}_{*} Y\right)\right)$, is also 0 , as desired.

Thus, we have proved by induction that $H_{*}\left(R\left(\mathcal{F}_{*} Y\right)\right)=0$, if $Y$ is finite.

Hence, for arbitrary $Y$, we have proved

$$
H_{*}\left(R\left(\mathcal{F}_{*} Y^{\prime}\right)\right)=0 \text { for all finite subsets } Y^{\prime} \text { of } Y .
$$

If $Y^{\prime} \subseteq Y^{\prime \prime}$ are finite subsets of $Y$, then there is a natural map

$$
R\left(\mathcal{F}_{*} Y^{\prime}\right) \rightarrow R\left(\mathcal{F}_{*} Y^{\prime \prime}\right)
$$

of differential graded $R$-bimodules, which is actually an embedding in all non-negative degrees. Hence we get a directed system

$$
\left(R\left(\mathcal{F}_{*} Y^{\prime}\right) \mid Y^{\prime} \text { a finite subset of } Y\right)
$$

of differential graded $R$-bimodules. We claim that

$$
\underset{\text { finite } Y^{\prime} \subseteq Y}{\lim } R\left(\mathcal{F}_{*} Y^{\prime}\right)=R\left(\mathcal{F}_{*} Y\right)
$$

By 3.1 (iii), $R(Y)$ is generated by the $R(y), y \in Y$, so

$$
\underset{\text { finite } Y_{Y^{\prime} \subseteq Y}}{\lim } R \otimes_{R\left(Y^{\prime}\right)} R=R \otimes_{R(Y)} R,
$$

so the degree -1 part of (7) holds. 
Since every element of $\mathcal{F}_{*} Y_{A}$ lies in $\mathcal{F}_{*} Y_{A}^{\prime}$ for some finite subset $Y^{\prime}$ of $Y$, we have $\underset{\text { finite } Y^{\prime} \subseteq Y}{\lim } \mathcal{F}_{*} Y_{A}^{\prime}=\mathcal{F}_{*} Y_{A}$, and (7) follows. Now

$$
\begin{aligned}
& H_{*}\left(R\left(\mathcal{F}_{*} Y\right)\right)=H_{*}\left(\lim _{\text {finite } Y^{\prime} \subseteq Y} R\left(\mathcal{F}_{*} Y^{\prime}\right)\right) \text {, by }(7), \\
& =\underset{\text { finite } Y^{\prime} \subseteq Y}{\lim _{*}} H_{*}\left(R\left(\mathcal{F}_{*} Y^{\prime}\right)\right)=0 \text {, by }(6) \text {. }
\end{aligned}
$$

This completes the proof.

We now want to show that all the other complexes defined above are acyclic. We shall make use of the following well-known fact.

4.5. Lemma. Suppose $\cdots \rightarrow C_{*, 2} \rightarrow C_{*, 1} \rightarrow C_{*, 0} \rightarrow C_{*,-1} \rightarrow 0$ is an exact sequence of differential $(\mathbb{N}-1)$-graded $R$-bimodules, such that, for all $j \in \mathbb{N}, C_{*, j}$ is acyclic. Then $C_{*,-1}$ is acyclic.

Proof: Let $j \in \mathbb{N}-1$.

Let $Z_{*, j}$ denote the image of $C_{*, j+1}$ in $C_{*, j}$; notice that $Z_{*,-1}=C_{*,-1}$. By the exactness of the given sequence, there is a short exact sequence of chain complexes $0 \rightarrow Z_{*, j+1} \rightarrow C_{*, j+1} \rightarrow Z_{*, j} \rightarrow 0$, and, since $C_{*, j+1}$ is acyclic, the resulting exact triangle for homology gives identifications $H_{i}\left(Z_{*, j}\right)=H_{i-1}\left(Z_{*, j+1}\right)$, for all $i \in \mathbb{Z}$. Let $n \in \mathbb{N}-1$, and apply the foregoing with $(i, j)=(n,-1),(n-1,0), \ldots,(-1, n)$, to obtain $H_{n}\left(Z_{*,-1}\right)=H_{n-1}\left(Z_{*, 0}\right)=\cdots=H_{-2}\left(Z_{*, n+1}\right)$. The final term, $H_{-2}\left(Z_{*, n+1}\right)$, is 0 , since the complexes are $\mathbb{N}-1$ graded. Thus the first term, $H_{n}\left(Z_{*,-1}\right)$, is 0 , for all $n \in \mathbb{N}-1$. Hence $Z_{*,-1}$ is acyclic, that is, $C_{*,-1}$ is acyclic.

We can now prove our main result.

4.6. Theorem. If Hypothesis 3.1 holds, and $Y$ is a subset of $X$, then $R\left(\mathcal{M}_{*} Y\right), R\left(\mathcal{I}_{*} Y\right)$, and $R\left(\mathcal{C}_{*} Y\right)$, are all acyclic.

Proof: Choose a total well-ordering $\prec$ of $\mathcal{M}_{0} Y_{A}$. There is a map $\mathcal{C}_{0} X_{A} \rightarrow \mathcal{M}_{0} X_{A}, X_{0} \mapsto \bar{X}_{0}$, where $\bar{X}_{0}$ is the least element of $\mathcal{M}_{0} X_{A}$, with respect to $\prec$, which contains $X_{0}$.

Consider any $m, n \in \mathbb{N}-1$.

Let $I(m, n)$ denote the set of all $m+n$-tuples $\left(X_{0}, \ldots, X_{m}, Y_{0}, \ldots, Y_{n}\right)$ such that the $Y_{j}$ lie in $\mathcal{M}_{0} Y_{A}$ with $Y_{0} \prec \cdots \prec Y_{n}$, and the $X_{i}$ lie in $\mathcal{C}_{0} Y_{A}$ with $X_{0} \subset \cdots \subset X_{m} \subseteq Y_{0} \cap \cdots \cap Y_{n}$. 
Let $C_{m, n}$ denote the $R$-bimodule

$$
\bigoplus_{\left(X_{0}, \ldots, X_{m}, Y_{0}, Y_{n}\right) \in I(m, n)} R \otimes_{R\left(X_{0}, \ldots, X_{m}, Y_{0}, \ldots, Y_{n}\right)} R
$$

where

$$
R\left(X_{0}, \ldots, X_{m}, Y_{0}, \ldots, Y_{n}\right)= \begin{cases}R\left(X_{0}\right) & \text { if } m \in \mathbb{N} \\ R\left(Y_{0} \cap \cdots \cap Y_{n}\right) & \text { if } m=-1, n \in \mathbb{N} \\ R(Y) & \text { if } m=-1, n=-1\end{cases}
$$

We abbreviate $a \otimes_{R\left(X_{0}, \ldots, X_{m}, Y_{0}, \ldots, Y_{n}\right)} b$ to $a\left\langle X_{0}, \ldots, X_{m} ; Y_{0}, \ldots, Y_{n}\right\rangle b$.

There is then a bi-graded $R$-bimodule $C_{*, *}=\underset{m \geq-1}{\bigoplus} \bigoplus_{n \geq-1} C_{m, n}$. We think of the indices $(m, n)$ as lying in the $m-n$ plane, largely in the first quadrant, and will speak of $m$ indexing the columns, and $n$ indexing the rows.

There are two commuting $R$-bimodule differentials $\partial_{x}, \partial_{y}: C_{*, *} \rightarrow C_{*, *}$ given by

$$
\begin{aligned}
\left(a \left\langleX_{0}, \ldots, X_{m} ; Y_{0}, \ldots,\right.\right. & \left.\left.Y_{n}\right\rangle b\right) \partial_{x} \\
& =\sum_{i=0}^{m}(-1)^{i} a\left\langle X_{0}, \ldots \hat{X}_{i} \ldots, X_{m} ; Y_{0}, \ldots, Y_{n}\right\rangle b, \\
\left(a \left\langleX_{0}, \ldots, X_{m} ; Y_{0}, \ldots,\right.\right. & \left.\left.Y_{n}\right\rangle b\right) \partial_{y} \\
& =\sum_{j=0}^{n}(-1)^{j} a\left\langle X_{0}, \ldots, X_{m} ; Y_{0}, \ldots \hat{Y}_{j} \ldots, Y_{n}\right\rangle b .
\end{aligned}
$$

It is straightforward to check that $\partial_{x}^{2}=\partial_{y}^{2}=0$, and $\partial_{x} \partial_{y}=\partial_{y} \partial_{x}$. We think of $\partial_{x}$ as acting on the $m$-co-ordinate, horizontally to the left, and $\partial_{y}$ as acting on the $n$-co-ordinate, vertically downwards. In summary, we have a large commuting diagram.

Notice that the row with index $n=-1$ agrees with $R\left(\mathcal{C}_{*} Y\right)$. Define $C_{*,+}=\bigoplus_{m \geq-1} \bigoplus_{n \geq 0} C_{m, n}$, and let $s_{x}: C_{*,+} \rightarrow C_{*,+}$ be given by

$$
\begin{aligned}
& \left(a\left\langle X_{0}, \ldots, X_{m} ; Y_{0}, \ldots, Y_{n}\right\rangle b\right) s_{x} \\
& \quad=(-1)^{m+1} a\left\langle X_{0}, \ldots, X_{m}, Y_{0} \cap \cdots \cap Y_{n} ; Y_{0}, \ldots, Y_{n}\right\rangle b
\end{aligned}
$$

where the result is understood to be 0 whenever $X_{m}=Y_{0} \cap \cdots \cap Y_{n}$. It is readily verified that $s_{x}$ is well-defined, and that $s_{x} \partial_{x}+\partial_{x} s_{x}$ acts as 
the identity on $C_{*,+}$. It follows that $H_{*}\left(C_{*,+}\right)=0$, which means that, for all $n \in \mathbb{N}$, the $n$th row is acyclic.

Notice that the column with index $m=-1$ agrees with $R\left(\mathcal{M}_{*} Y\right)$. Define $C_{+, *}=\bigoplus_{m \geq 0} \bigoplus_{n \geq-1} C_{m, n}$, and let $s_{y}: C_{+, *} \rightarrow C_{+, *}$ be given by

$$
\left(a\left\langle X_{0}, \ldots, X_{m} ; Y_{0}, \ldots, Y_{n}\right\rangle b\right) s_{y}=a\left\langle X_{0}, \ldots, X_{m} ; \bar{X}_{m}, Y_{0}, \ldots, Y_{n}\right\rangle b
$$

where the result is understood to be 0 whenever $\bar{X}_{m}=Y_{0}$. It is readily verified that $s_{x}$ is well-defined, and that $s_{y} \partial_{y}+\partial_{y} s_{y}$ acts as the identity on $C_{+, *}$. It follows that, for all $m \in \mathbb{N}$, the $m$ th column is acyclic.

We can interchange $m$ and $n$, and apply Lemma 4.5 , to deduce that, if $R\left(\mathcal{M}_{*} Y\right)$ is acyclic, then $R\left(\mathcal{C}_{*} Y\right)$ is acyclic also.

If we consider the commuting sub-diagram obtained by reducing $\mathcal{C}_{0} Y_{A}$ to $\mathcal{I}_{0} Y_{A}$ (or, indeed, to any set between $\mathcal{I}_{0} Y_{A}$ and $\mathcal{C}_{0} Y_{A}$ ) throughout, then the maps $s_{x}$ and $s_{y}$ act on the sub-diagram, and we again deduce that if $R\left(\mathcal{M}_{*} Y\right)$ is acyclic, then $R\left(\mathcal{I}_{*} Y\right)$ is acyclic.

Now consider the commuting sub-diagram obtained by reducing $\mathcal{C}_{0} Y_{A}$ to $\mathcal{F}_{0} Y_{A}$ throughout. Here $s_{y}$ acts on the sub-diagram, so the columns with non-negative index are acyclic. If any element of $\mathcal{M}_{0} Y_{A}$ is infinite, then $s_{x}$ does not act on the sub-diagram. However, the rows in the sub-diagram are easily seen to consist of direct sums of copies of the complexes $R\left(\mathcal{F}_{*} W\right)$, where $W$ ranges over the family consisting of $Y$ and sets of the form $Y_{0} \cap \cdots \cap Y_{n}$, with $Y_{0} \prec \cdots \prec Y_{n}$ in $\mathcal{M}_{0} Y_{A}$. We proved, in Theorem 4.4, that all such complexes $R\left(\mathcal{F}_{*} W\right)$ are acyclic. Thus all the rows of the sub-diagram are acyclic, so, by Lemma 4.5, $R\left(\mathcal{M}_{*} Y\right)$ is acyclic. Now, by the two preceding paragraphs, we see that $R\left(\mathcal{C}_{*} Y\right)$ and $R\left(\mathcal{I}_{*} Y\right)$ are acyclic also.

4.7. Remarks. There is a certain topological flavour to the above proof, and it is interesting to identify the sources.

(i) The essence of the argument used in proving Theorem 4.6 may be viewed as an extension to non-constant coefficient systems of a theorem in simplicial homology proved by A. Weil [12], who also attributes a similar result to Leray.

If an augmented simplicial complex $\mathcal{S}_{*}$ is a union of subcomplexes $\mathcal{S}_{*}^{(i)}$, the nerve of this covering is the simplicial complex with vertices the $\mathcal{S}_{*}^{(i)}$, and simplices the finite collections with non-empty intersection.

The main result of section 3 of [12] is that, if each non-empty intersection of the $\mathcal{S}_{*}^{(i)}$ is acyclic, then the homology (with constant coefficients) of the nerve of the covering is isomorphic to the homology of $\mathcal{S}_{*}$. 
To state the generalization of this to non-constant coefficients, it is more convenient to use (non-augmented) simplicial complexes, because it is no longer clear what coefficient object should be assigned to the simplex of dimension -1 .

Given a poset $\left(\mathcal{P}_{0}, \prec\right)$, and a functor $F$ from $\mathcal{P}_{0}$ to an abelian category, one may define a chain complex with $F$-coefficients for the complex $\mathcal{P}_{*+}$, where the simplex $\left\{p_{0}, \ldots, p_{n}\right\}$, with $p_{0} \prec \cdots \prec p_{n}$, is assigned coefficient object $F\left(p_{0}\right)$. The homology of this chain complex, denoted $H_{*}\left(\mathcal{P}_{*+}, F\right)$, is called the homology of $\mathcal{P}_{*+}$ with coefficients in $F$. If $F$ is a constant functor, then this is just the ordinary homology of $\mathcal{P}_{*_{+}}$. If $\left(\mathcal{P}_{0}, \prec\right)$ is a union of sub-posets $\left(\mathcal{P}_{0}^{(i)}, \prec\right)$, such that every chain in $\mathcal{P}_{0}$ is contained in one of the $\mathcal{P}_{0}^{(i)}$, then the simplicial complex $\mathcal{P}_{*+}$ is equal to the union of the subcomplexes $\mathcal{P}_{*+}^{(i)}$. If each non-empty intersection of the posets $\left(\mathcal{P}_{0}^{(i)}, \prec\right)$ is $F$-acyclic (in the sense that if $\left(\mathcal{Q}_{0}, \prec\right)$ is such an intersection, then $H_{i}\left(\mathcal{Q}_{*+}, F\right)=0$ for $\left.i>0\right)$, then an argument similar to that given by Weil shows that, even in this generality, the homology of $\mathcal{P}_{*+}$ with coefficients in $F$ may be calculated as the homology of a chain complex associated to the nerve of the covering. This chain complex associates to each non-empty intersection $\left(\mathcal{Q}_{0}, \prec\right)$ of the $\left(\mathcal{P}_{0}^{(i)}, \prec\right)$, the coefficient object $H_{0}\left(\mathcal{Q}_{*+}, F\right)$.

- We shall not prove the above result in its full generality here, because we do not use it, and also because three special cases of it appear in our proof of Theorem 4.6. We may define a functor from $\mathcal{C}_{0} Y_{A}$ (resp. $\mathcal{F}_{0} Y_{A}$, $\mathcal{I}_{0} Y_{A}$ ) to $R$-bimodules, which sends the subset $Z$ to $R \otimes_{R(Z)} R$. The corresponding chain complex is then $R\left(\mathcal{C}_{*_{+}} Y\right)$ (resp. $R\left(\mathcal{F}_{*+} Y\right), R\left(\mathcal{I}_{*_{+}} Y\right)$ ). Any chain in $\mathcal{C}_{0} Y_{A}$ (resp. $\mathcal{F}_{0} Y_{A}, \mathcal{I}_{0} Y_{A}$ ) is contained in $\mathcal{C}_{0} Z_{A}$ (resp. $\mathcal{F}_{0} Z_{A}$, $\left.\mathcal{I}_{0} Z_{A}\right)$ for some $Z \in \mathcal{M}_{0} Y_{A}$. Thus we may view $\mathcal{M}_{*+} Y_{A}$ as the nerve of the covering of $\mathcal{C}_{*_{+}} Y_{A}$ (resp. $\mathcal{F}_{*_{+}} Y_{A}, \mathcal{I}_{*_{+}} Y_{A}$ ) by its subcomplexes which come from maximal complete subgraphs. Since an intersection of complete graphs is a complete graph, we may apply the generalization of Weil's theorem, once we have proved that for any complete graph $Y$ the chain complex $R\left(\mathcal{C}_{*_{+}} Y\right)$ (resp. $R\left(\mathcal{F}_{*_{+}} Y\right), R\left(\mathcal{I}_{*_{+}} Y\right)$ ) is acyclic. For the case of $R\left(\mathcal{F}_{*+} Y\right)$ this was done in the proof of Theorem 4.4. The proof of the claim for $R\left(\mathcal{C}_{*+} Y\right)$ and $R\left(\mathcal{I}_{*+} Y\right)$ is contained in the proof of Theorem 4.6.

The latter proof generalizes to arbitrary functors $F$ as follows. If $\mathcal{P}_{0}$ is any poset with a greatest element $p$, and $F$ is any functor from $\mathcal{P}_{0}$ to an abelian category, then it is easily verified that

$$
H_{n}\left(\mathcal{P}_{*+}, F\right)= \begin{cases}F(p) & \text { if } n=0 \\ 0 & \text { if } n \neq 0\end{cases}
$$


On the other hand, the proof of acyclicity of $R\left(\mathcal{F}_{*} Y\right)$ relies on the fact that $R\left(\mathcal{F}_{*}-\right)$ preserves direct limits, so does not generalize to arbitrary functors $F$.

(ii) M.W. Davis used finitely generated Coxeter groups in [8] to construct interesting contractible $C W$-complexes. The barycentric subdivisions of his complexes give acyclic augmented chain complexes which are closely related to the acyclic complexes of the form $R\left(\mathcal{C}_{*} X\right)$; the two complexes are basically the same in the case of integral group rings of finitely generated right-angled Coxeter groups.

We now deduce consequences about projective dimensions.

4.8. Corollary. Suppose that Hypothesis 3.1 holds. Then, for any left $R$-module $M$, there is an exact sequence of left $R$-modules

$$
\begin{aligned}
\cdots \rightarrow X_{X_{0} \subset \cdots \subset X_{n} \text { in } \mathcal{I}_{0}\left(X_{A}\right)}^{\oplus} R \otimes_{R\left(X_{0}\right)} M \rightarrow \cdots \\
\cdots \rightarrow \underset{X_{0} \in \mathcal{I}_{0}\left(X_{A}\right)}{\oplus} R \otimes_{R\left(X_{0}\right)} M \rightarrow M \rightarrow 0,
\end{aligned}
$$

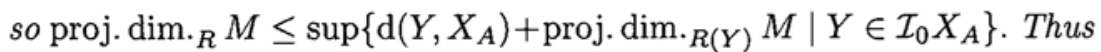
l. gl. $\operatorname{dim} . R \leq \sup \left\{\mathrm{d}\left(Y, X_{A}\right)+\right.$ l.gl. $\left.\operatorname{dim} . R(Y) \mid Y \in \mathcal{I}_{0} X_{A}\right\}$.

Here proj. dim. ${ }_{R(Y)} M$ denotes the minimum of the lengths of projective $R(Y)$-resolutions of $M$, and l.gl. $\operatorname{dim} . R(Y)$ denotes the supremum of the projective dimensions of left $R(Y)$-modules.

Proof: We have the resolution (1), by Theorem 4.6. All the terms of (1) are flat as right $R$-modules, so (1) remains exact under $-\otimes_{R} M$, giving us the exact sequence (8).

Consider any $X_{0} \in \mathcal{I}_{0} X_{A}$. Since $R$ is flat as right $R\left(X_{0}\right)$-module, any projective $R\left(X_{0}\right)$-resolution of $M$ lifts, under $R \otimes_{R\left(X_{0}\right)}$, to a projective $R$-resolution of $R \otimes_{R\left(X_{0}\right)} M$.

Thus we get certain projective $R$-resolutions of all the terms of the sequence (8), and these can be used to construct a double complex. The corresponding total complex is then a projective $R$-resolution of $M$, whose length is

$$
\sup \left\{n+\text { proj. } \operatorname{dim}_{R_{\left(X_{0}\right)}} M \mid X_{0} \subset \cdots \subset X_{n} \text { in } \mathcal{I}_{0} X_{A}\right\},
$$

and this gives the desired bounds.

The main motivation for our work was the result of Bergman [3], that, if Hypothesis 3.1 holds and $X_{A}$ is finite, then

l. gl. $\operatorname{dim} . R \leq \sup \left\{\left|Y^{\prime}-Y\right|+\right.$ l.gl. $\operatorname{dim} . R(Y) \mid Y \subseteq Y^{\prime}$ in $\left.\mathcal{C}_{0} X_{A}\right\}$; 
this implication follows from Corollary 4.8, or even from Theorem 4.4 together with the argument used in the proof of Corollary 4.8.

Let us record some of the consequences for the special case of graph products of groups.

4.9. Corollary. Let $(G(x) \mid x \in X)$ be a family of groups indexed by $X$, and let $G=G\left(X_{A}\right)$. Then there there is an exact sequence of left $K G$-modules

$$
\begin{aligned}
\cdots \rightarrow \underset{X_{0} \subset \cdots \subset X_{n} \text { in } \mathcal{I}_{0}\left(X_{A}\right)}{\oplus} K\left[G / G\left(X_{0}\right)\right] \rightarrow \ldots \\
\cdots \rightarrow \underset{X_{0} \in \mathcal{I}_{0}\left(X_{A}\right)}{\oplus} K\left[G / G\left(X_{0}\right)\right] \rightarrow K \rightarrow 0 .
\end{aligned}
$$

Hence $\operatorname{cd}_{K} G\left(X_{A}\right) \leq \sup \left\{\mathrm{d}\left(Y, X_{A}\right)+\operatorname{cd}_{K} G\left(Y_{A}\right) \mid Y \in \mathcal{I}_{0} X_{A}\right\}$.

Proof: This is the case of Corollary 4.8 where $R(x)=K[G(x)]$, for all $x \in X$, and $M=K_{\varepsilon}$, where $K_{\varepsilon}$ denotes the left $K$-module $K$ made into a left $K\left[G\left(X_{A}\right)\right]$-module with trivial $G\left(X_{A}\right)$-action.

\section{Refining resolutions}

This section is devoted to describing how the resolution (1) given by Theorem 4.6 can be refined, by choosing a useful sub-resolution with split exact quotient.

Throughout this section, let us suppose that Hypothesis 3.1 holds, so $k$ is the principal ideal domain chosen in $3.1(v i)$.

5.1. Definition. Let $D_{*}$ be a differential graded $R$-bimodule with differential $\partial_{*}$.

Suppose we have a decomposition of differential graded $R$-bimodules $D_{*}=D_{*, 1} \oplus D_{*, 2}$, and that $D_{*, 1}$ is split, which means that there exists a graded $R$-bimodule endomorphism $s_{*}$ of $D_{*, 1}$ of degree +1 , such that $s_{*} \partial_{*}+\partial_{*} s_{*}=1$ on $D_{*, 1}$; we have already used.such maps to good advantage, in the proofs of Theorems 4.4 and 4.6.

Here $s_{*} \partial_{*} s_{*} \partial_{*}=s_{*} \partial_{*}\left(1-\partial_{*} s_{*}\right)=s_{*} \partial_{*}-s_{*} \partial_{*} \partial_{*} s_{*}=s_{*} \partial_{*}+0=s_{*} \partial_{*}$. Thus $s_{*} \partial_{*}$ is idempotent, and so is $\partial_{*} s_{*}=1-s_{*} \partial_{*}$. In particular, $s_{*} \partial_{*}$ and $\partial_{*} s_{*}$ commute, and have product 0 , so $\left(s_{*} \partial_{*} s_{*}\right)^{2}=0$.

Now $1=s_{*} \partial_{*}+\partial_{*} s_{*}=s_{*} \partial_{*} s_{*} \partial_{*}+\partial_{*} s_{*} \partial_{*} s_{*}=\left(s_{*} \partial_{*} s_{*}\right) \partial_{*}+\partial_{*}\left(s_{*} \partial_{*} s_{*}\right)$. Thus we can replace $s_{*}$ with $s_{*} \partial_{*} s_{*}$, and so assume that $s_{*} \partial_{*} s_{*}=s_{*}$, and that $s_{*}^{2}=0$. 
Let us extend $s_{*}$ to all of $D_{*}$ by specifying that $D_{*, 2} s_{*}=0$. Then $s_{*}$ is an $R$-bimodule endomorphism of $D_{*}$, of degree +1 , such that $s_{*}^{2}=0$, $s_{*} \partial_{*} s_{*}=s_{*}, D_{*, 1}=D_{*}\left(s_{*} \partial_{*}+\partial_{*} s_{*}\right)$, and $D_{*, 2}=D_{*}\left(1-s_{*} \partial_{*}-\partial_{*} s_{*}\right)$.

An endomorphism $s_{*}$ of degree +1 of a differential graded $R$-bimodule such that $s_{*}^{2}=0$ and $s_{*} \partial_{*} s_{*}=s_{*}$ will be called a component-contracting homotopy. In this event, $e=s_{*} \partial_{*}+\partial_{*} s_{*}$ is idempotent, and, as differential graded $R$-bimodule, $D_{*}$ decomposes as $D_{*} e \oplus D_{*}(1-e)$, such that $D_{*} e$ is split, with contracting homotopy $s_{*}$.

5.2. Definition. Let $M$ be a $k$-module. The rank of $M$ over $k$, denoted $\mathrm{rk}_{k} M$, is the minimum of the cardinals of the $k$-generating sets of $M$. The relation rank of $M$ over $k$, denoted $\operatorname{rel}^{-\mathrm{rk}_{k}} M$, is the minimum of the ranks of kernels of surjective $k$-linear maps from free $k$-modules to $M$. By a minimal presentation of $M$ we mean a short exact sequence of $k$-modules, $0 \rightarrow G \rightarrow F \rightarrow M \rightarrow 0$, with $F, G$ free, such that rel-rk $k=\mathrm{rk}_{k} G$. In this event it is easy to see that $\operatorname{rk}_{k} M=\mathrm{rk}_{k} F$.

The following is well-known.

5.3. Lemma. Let $k$ be a principal ideal domain. For any exact sequence of $k$-modules $0 \rightarrow G \rightarrow F \rightarrow M \rightarrow 0$, such that $G \subseteq F$ are free $k$-modules, there exist decompositions $F=H \oplus F^{\prime}, G=H \oplus G^{\prime}$

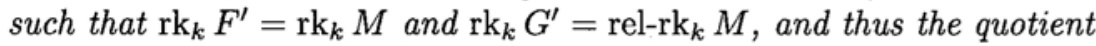
presentation $0 \rightarrow G^{\prime} \rightarrow F^{\prime} \rightarrow M \rightarrow 0$ is minimal.

Proof: Let $0 \rightarrow A \rightarrow B \rightarrow M \rightarrow 0$ be any minimal presentation of $M$, with $A \subseteq B$ free. Since $F$ is free, there is a map $F \rightarrow B$ such that

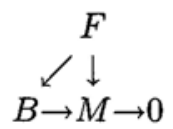

commutes. Let $B^{\prime}$ be the image of $F \rightarrow B$, so $B^{\prime}$ is free, and $B^{\prime} \rightarrow M$ is surjective. Let $A^{\prime}$ be the kernel of $B^{\prime} \rightarrow M$, so $A^{\prime} \subseteq A$, so the rank of $A^{\prime}$ is at most the rank of $A$, so $0 \rightarrow A^{\prime} \rightarrow B^{\prime} \rightarrow M \rightarrow 0$ is also a minimal presentation. Now

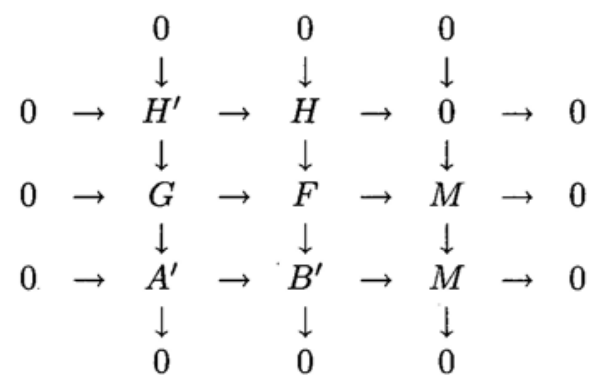


is a commuting diagram with exact columns and rows. Hence $H^{\prime}=H$. Since the middle column splits, $H$ has the desired properties.

5.4. Definition. Let $D_{*}$ be a differential graded $k$-module that is free as $k$-module.

We now define a non-canonical component-contracting homotopy $s_{*}$ which reduces $\mathrm{rk}_{k} D_{*}$ as much as possible; in particular, $s_{*}$ reduces $D_{n}$ to 0 whenever $H_{n}\left(D_{*}\right)=0$ and $H_{n-1}\left(D_{*}\right)$ is free, as $k$-module.

Let $n$ be an integer.

We have a presentation $0 \rightarrow \operatorname{Im} \partial_{n+1} \rightarrow \operatorname{Ker} \partial_{n} \rightarrow H_{n}\left(D_{*}\right) \rightarrow 0$.

By Lemma 5.3 we can choose decompositions

$$
\operatorname{Ker} \partial_{n}=A_{n} \oplus X_{n}, \operatorname{Im} \partial_{n+1}=A_{n} \oplus Y_{n}, Y_{n} \subseteq X_{n},
$$

such that $\operatorname{rk}_{k} X_{n}=\operatorname{rk}_{k} H_{n}\left(D_{*}\right), \operatorname{rk}_{k} Y_{n}=\operatorname{rel}^{-\operatorname{rk}_{k}} H_{n}\left(D_{*}\right)$, and we then have a minimal presentation

$$
0 \rightarrow Y_{n} \stackrel{i_{n}}{\longrightarrow} X_{n} \rightarrow H_{n}\left(D_{*}\right) \rightarrow 0,
$$

where $i_{n}$ is the inclusion map.

Since $D_{n-1}$ is a free $k$-module, the image of $\partial_{n}: D_{n} \rightarrow D_{n-1}$ is a free $k$-module, so we may choose a $k$-linear isomorphism

$$
D_{n} \approx \operatorname{Ker} \partial_{n} \oplus \operatorname{Im} \partial_{n}=A_{n} \oplus X_{n} \oplus A_{n-1} \oplus Y_{n-1} .
$$

Thus we have a commuting diagram

$$
\begin{gathered}
D_{n+1} \stackrel{\sim}{\longrightarrow} A_{n+1} \oplus X_{n+1} \oplus A_{n} \oplus Y_{n} \\
\downarrow \partial_{n+1} \\
\qquad\left(\begin{array}{llll}
0 & 0 & 0 & 0 \\
0 & 0 & 0 & 0 \\
1 & 0 & 0 & 0 \\
0 & i_{n} & 0 & 0
\end{array}\right) \\
D_{n} \stackrel{\sim}{\longrightarrow} A_{n} \oplus X_{n} \oplus A_{n-1} \oplus Y_{n-1} .
\end{gathered}
$$

Let $s_{n}: D_{n} \rightarrow D_{n+1}$ be the map in the reverse direction that corresponds to

$$
\left(\begin{array}{llll}
0 & 0 & 1 & 0 \\
0 & 0 & 0 & 0 \\
0 & 0 & 0 & 0 \\
0 & 0 & 0 & 0
\end{array}\right) .
$$

It is clear that $s_{n} s_{n+1}=0$ and $s_{n} \partial_{n+1} s_{n}=s_{n}$, so $s_{*}$ is a component-contracting homotopy, and $H_{*}\left(D_{*}\right) \approx H_{*}\left(D_{*}\left(1-s_{*} \partial_{*}-\partial_{*} s_{*}\right)\right)$, as graded $k$-modules.

Although $s_{*}$ depends on the choice of the decompositions, there is at least one invariant, namely the graded $k$-module isomorphism class of 
$D_{*}\left(1-s_{*} \partial_{*}-\partial_{*} s_{*}\right)$, since $D_{n}\left(1-s_{n} \partial_{n+1}-\partial_{n} s_{n-1}\right) \approx X_{n} \oplus Y_{n-1}$, so $\mathrm{rk}_{k} D_{n}\left(1-s_{n} \partial_{n+1}-\partial_{n} s_{n-1}\right)=\operatorname{rk}_{k} H_{n}\left(D_{*}\right)+\operatorname{rel}^{-\mathrm{rk}_{k}} H_{n-1}\left(D_{*}\right)$.

Notice that this is 0 if and only if $H_{n}\left(D_{*}\right)=0$ and $H_{n-1}\left(D_{*}\right)$ is free, as $k$-module.

5.5. Notation. For any $Y \in \mathcal{I}_{0} X_{A}$, and $n \in \mathbb{N}$, we define

$$
c_{n}\left(Y, X_{A}, k\right)=\operatorname{rk}_{k} H_{n-1}\left(\mathcal{I}_{*}\left(Y, X_{A}\right), k\right)+\operatorname{rel}^{-\operatorname{rk}_{k}} H_{n-2}\left(\mathcal{I}_{*}\left(Y, X_{A}\right), k\right) .
$$

5.6. Theorem. If Hypothesis 3.1 holds, and $X_{A}$ is finite-dimensional, then there exists a finite exact sequence of $R$-bimodules

$$
\begin{aligned}
\cdots \rightarrow \underset{Y \in \mathcal{I}_{0} X_{A}}{\oplus} c_{n, Y} R \otimes_{R(Y)} R & \rightarrow \ldots \\
\cdots & \rightarrow \underset{Y \in \mathcal{I}_{0} X_{A}}{\oplus} c_{0, Y} R \otimes_{R(Y)} R \rightarrow R \rightarrow 0,
\end{aligned}
$$

where $c_{n, Y} R \otimes_{R(Y)} R$ denotes a direct sum of $c_{n}\left(Y, X_{A}, k\right)$ copies of $R \otimes_{R(Y)} R$.

The maps in (9) have not been specified, nor are they in any way canonical. There is an underlying exact sequence of $k$-modules obtained by taking all the $R(x)$ to be $k$. For $k=\mathbb{Z}$, the underlying exact sequence is the augmented chain complex of some acyclic CW-complex, provided the resulting map $\underset{Y \in \mathcal{I}_{0} X_{A}}{\oplus} c_{1, Y} \mathbb{Z} \rightarrow \underset{Y \in \mathcal{I}_{0} X_{A}}{\oplus} c_{0, Y} \mathbb{Z}$ behaves like a boundary map for edges, that is, sends each of the given generators of the domain to the difference of two of the given generators of the codomain.

Proof: Recall that the augmented complex $R\left(\mathcal{I}_{*} X\right)=\underset{n \geq-1}{\oplus} R\left(\mathcal{I}_{n} X_{A}\right)$ presented in Definition 4.2, is acyclic, by Theorem 4.6.

Let $D_{*}$ denote the graded $R$-sub-bimodule $\bigoplus_{n \geq 0} R\left(\mathcal{I}_{n} X_{A}\right)$ of $R\left(\mathcal{I}_{*} X\right)$, endowed with the natural induced differential $\partial_{*}$; thus $D_{*}$ is actually a quotient complex of $R\left(\mathcal{I}_{*} X_{A}\right)$, and its homology is $R$, concentrated in degree 0 .

For the reminder of the proof, for reasons of typographical aesthetics, we supress most of the $*$ subscripts.

We want to apply a component-contracting homotopy to $D$ to eliminate redundancies.

For each $Y \in \mathcal{I}_{0} X_{A}$, let $D_{Y}$ (resp. $D_{Y}^{\prime}$ ) denote the graded $R$-subbimodule (resp. $k$-submodule) of $D$ generated by those $\left\langle X_{0}, \ldots, X_{n}\right\rangle$ for which $X_{0}=Y$. Thus we can identify $D_{Y}=\left(R \otimes_{R(Y)} R\right) \otimes_{k} D_{Y}^{\prime}$. 
Then, as graded $R$-bimodule, $D=\bigoplus_{Y \in \mathcal{I}_{0} X_{A}} D_{Y}$.

The differential $\partial$ has a corresponding componentwise decomposition as $\partial=\prod_{Y \in \mathcal{I}_{0}\left(X_{A}\right)} \sum_{Y^{\prime} \in \mathcal{I}_{0}\left(X_{A}\right)} \partial_{Y, Y^{\prime}}$, where $\partial_{Y, Y^{\prime}}$ is an $R$-bimodule map from $D_{Y}$ to $D_{Y^{\prime}}$. From the definition of $\partial$, we know that $\partial_{Y, Y^{\prime}}=0$ unless $Y \subseteq Y^{\prime}$.

Again for aesthetic reasons, we supress the product symbol in the preceding expression, and write simply $\partial=\sum_{Y \subseteq Y^{\prime} \text { in } \mathcal{I}_{0}\left(X_{A}\right)} \partial_{Y, Y^{\prime}}$.

For any $Y^{\prime} \subseteq Y^{\prime \prime}$ in $\mathcal{I}_{0} X_{A}$, the $\left(Y^{\prime}, Y^{\prime \prime}\right)$-component of $\partial^{2}=0$ is $\sum_{Y^{\prime} \subseteq Y \subseteq Y^{\prime \prime}} \partial_{Y^{\prime}, Y} \partial_{Y, Y^{\prime \prime}}=0$.

Thus, for any $Y \in \mathcal{I}_{0} X_{A}, \partial_{Y, Y}^{2}=0$, so $D_{Y, Y}$ (resp. $D_{Y, Y}^{\prime}$ ) is a differential graded $R$-bimodule (resp. $k$-module) with differential $\partial_{Y, Y}$.

Observe that $D_{Y, Y}^{\prime}$ is isomorphic to the chain complex of the augmented simplicial complex $\mathcal{I}_{*}\left(Y, X_{A}\right)$ with coefficients in $k$, but with the degree of the latter shifted up by 1 , so an $n$-simplex $\left\{X_{0}, \ldots, X_{n}\right\}$ in $\mathcal{I}_{n}\left(Y, X_{A}\right)$ corresponds to a generator $(-1)^{n}\left\langle Y, X_{0}, \ldots, X_{n}\right\rangle$ of $D_{Y, Y}^{\prime}$ of degree $n+1$, for all $n \in \mathbb{N}-1$. Hence $H_{n}\left(\mathcal{I}_{*}\left(Y, X_{A}\right), k\right) \approx H_{n+1}\left(D_{Y, Y}^{\prime}\right)$.

As in Definition 5.4, we can choose a component-contracting homotopy $s_{Y}$ of $D_{Y}^{\prime}$ such that $D_{n, Y}^{\prime}\left(1-s_{n, Y} \partial_{n+1, Y, Y}-\partial_{n, Y, Y} s_{n-1, Y}\right)$ is $k$-free of rank

$$
\begin{aligned}
\operatorname{rk}_{k} H_{n}\left(D_{Y}^{\prime}\right)+\operatorname{rel}_{-\mathrm{rk}_{k}} H_{n-1}\left(D_{Y}^{\prime}\right) & \\
& =\operatorname{rk}_{k} H_{n-1}\left(\mathcal{I}_{*}\left(Y, X_{A}\right), k\right)+\operatorname{rel-rk}_{k} H_{n-2}\left(\mathcal{I}_{*}\left(Y, X_{A}\right), k\right) \\
& =c_{n}\left(Y, X_{A}, k\right)=c_{n, Y}
\end{aligned}
$$

It is a simple matter to lift $s_{Y}$ to a degree $+1, R$-bimodule endomorphism on all of $D_{Y}=\left(R \otimes_{R(Y)} R\right) \otimes_{k} D_{Y, Y}^{\prime}$, and we again use $s_{Y}$ to denote the lifted map. As $R$-bimodule,

$$
D_{n, Y}\left(1-s_{n, Y} \partial_{n+1, Y, Y}-\partial_{n, Y, Y} s_{n-1, Y}\right) \approx c_{n, Y} R \otimes_{R(Y)} R .
$$

We now express $\partial$ as a sum of two degree $-1, R$-bimodule endomorphisms of $D$. Let $\partial^{=}$denote $\sum_{Y \in \mathcal{I}_{0} X_{A}} \partial_{Y, Y}$, and let $\partial^{+}$denote $\partial-\partial^{=}$, so $\partial^{+}=\sum_{Y \subset Y^{\prime} \text { in } \mathcal{I}_{0}\left(X_{A}\right)} \partial_{Y, Y^{\prime}}$.

Let $s^{=}=\sum_{Y \in \mathcal{I}_{0} X_{A}} s_{Y}$, a degree $+1, R$-bimodule endomorphism of $D$. Notice that $s^{=} s^{=}=0$, and $s^{=\partial^{=}} s^{=}=s^{=}$.

Now $1+s^{=} \partial^{+}$is a degree $0, R$-bimodule endomorphism of $D$, and we claim it is an automorphism. Let $d=1+\operatorname{dim} \mathcal{I}_{*} X_{A}$, which is finite by 
hypothesis. Then the depth with respect to $X_{A}$ of each element of $\mathcal{I}_{0} X_{A}$ is at most $d$, and at least 0 . Since

$$
\left(D_{Y}\right) s^{=} \partial^{+} \subseteq\left(D_{Y}\right) \partial^{+} \subseteq \underset{Y^{\prime} \supset Y}{\bigoplus} D_{Y^{\prime}}
$$

we see that $s^{=} \partial^{+}$is strictly decreasing with respect to depth. It follows that $\left(s^{=} \partial^{+}\right)^{d+1}=0$. Hence $1+s^{=} \partial^{+}$is an automorphism of $D_{Y}$, with inverse $1-s^{=} \partial^{+}+\left(s^{=} \partial^{+}\right)^{2}+\cdots+\left(-s^{=} \partial^{+}\right)^{d}$, as claimed.

Define $s=\left(1+s^{=} \partial^{+}\right)^{-1} s^{=}=s^{=}\left(1+\partial^{+} s^{=}\right)^{-1}$, so $s$ is a degree +1 , $R$-bimodule endomorphism of $D$.

To see that $s$ is a component-contracting homotopy of $D$ observe that:

$$
s s=\left(1+s^{=} \partial^{+}\right)^{-1} s^{=} s^{=}\left(1+\partial^{+} s^{=}\right)^{-1}=0 ;
$$

$$
\begin{aligned}
s \partial s & =\left(1+s^{=} \partial^{+}\right)^{-1} s^{=}\left(\partial^{=}+\partial^{+}\right) s^{=}\left(1+\partial^{+} s^{=}\right)^{-1} \\
& =\left(1+s^{=} \partial^{+}\right)^{-1} s^{=} \partial^{=} s^{=}\left(1+\partial^{+} s^{=}\right)^{-1}+\left(1+s^{=} \partial^{+}\right)^{-1} s^{=} \partial^{+} s^{=}\left(1+\partial^{+} s^{=}\right)^{-1} \\
& =\left(1+s^{=} \partial^{+}\right)^{-1} s^{=}\left(1+\partial^{+} s^{=}\right)^{-1}+\left(1+s^{=} \partial^{+}\right)^{-1} s^{=} \partial^{+} s^{=}\left(1+\partial^{+} s^{=}\right)^{-1} \\
& =\left(1+s^{=} \partial^{+}\right)^{-1} s^{=}\left(1+\partial^{+} s^{=}\right)\left(1+\partial^{+} s^{=}\right)^{-1}=\left(1+s^{=} \partial^{+}\right)^{-1} s^{=}=s .
\end{aligned}
$$

Write $e=1-s \partial-\partial s$ and $e^{=}=1-s^{=} \partial^{=}-\partial^{=} s^{=}$. Then $e, e^{=}$ are idempotent, degree $0, R$-bimodule endomorphisms of $D$, which are decreasing with respect to depth. Moreover $e-e^{=}$is strictly decreasing with respect to depth. Let $u=e e^{=}+(1-e)\left(1-e^{=}\right)$, a degree $0, R$-bimodule endomorphism of $D$. Since

$$
1-u=e+e^{=}-2 e e^{=}=e\left(e-e^{=}\right)+\left(e-e^{=}\right) e^{=}
$$

is strictly decreasing with respect to depth, we see $(1-u)^{d+1}=0$, so $u$ is an automorphism, with inverse $1+(1-u)+(1-u)^{2}+\cdots+(1-u)^{d}$. Also $e u=e e^{=}+0=u e^{=}$, so $D e \approx D e u=D u e^{=}=D e^{=}$. That is, as $R$-bimodules,

$$
\begin{aligned}
D_{n}\left(1-s_{n} \partial_{n+1}-\partial_{n} s_{n-1}\right) & \approx D_{n}\left(1-s_{n}^{=} \partial_{n+1}^{=}-\partial_{n}^{=} s_{n-1}^{=}\right) \\
& \approx \bigoplus_{Y \in \mathcal{I}_{0} X_{A}} c_{n, Y} R \otimes_{R(Y)} R .
\end{aligned}
$$

Moreover, $H_{*}\left(D_{*}(1-s \partial-\partial s)\right) \approx H_{*}\left(D_{*}\right)$, which is $R$, concentrated in degree 0 . This gives an exact sequence (9).

The resolution (9) can be expressed in the notation introduced in Example 2.3, entirely in terms of the graph $X_{A}$, and the principal ideal domain $k$, so, in this sense, (9) is independent of the choice of rings $K$ and $R(x), x \in X$.

In the same way that Corollary 4.8 was deduced from Theorem 4.6, the following can be deduced from Theorem 5.6. 
5.7. Corollary. Suppose that Hypothesis 3.1 holds, and that $X_{A}$ is finite-dimensional. Then, for any left $R$-module $M$, there is a finite exact sequence of left $R$-modules

$$
\begin{aligned}
\cdots \rightarrow \underset{Y \in \mathcal{I}_{0} X_{A}}{\oplus} c_{n, Y} R \otimes_{R(Y)} M & \rightarrow \ldots \\
\cdots & \rightarrow \underset{Y \in \mathcal{I}_{0} X_{A}}{\oplus} c_{0, Y} R \otimes_{R(Y)} M \rightarrow M \rightarrow 0,
\end{aligned}
$$

so proj. dim. $._{R} M \leq \sup \left\{\operatorname{hd}_{k}\left(Y, X_{A}\right)+\right.$ proj.dim. $\left.{ }_{R(Y)} M \mid Y \in \mathcal{I}_{0} X_{A}\right\}$. Thus 1. gl. dim. $R \leq \sup \left\{\operatorname{hd}_{k}\left(Y, X_{A}\right)+\right.$ l.gl. dim. $\left.R(Y) \mid Y \in \mathcal{I}_{0} X_{A}\right\}$.

We now illustrate why omitting the hypothesis of finite-dimensionality would invalidate Theorem 5.6 and Corollary 5.7.

5.8. Example. Let $X$ be the following set of sub-intervals of the interval $[0,1]$ :

$$
\left\{\left[\frac{i}{2^{n}}, \frac{i+1}{2^{n}}\right] \mid i, n \in \mathbb{N}, i+1 \leq 2^{n}\right\} \cup\{\{y\} \mid y \in[0,1]\} .
$$

We will construct a graph having vertex set $X$, but let us first make some observations.

Notice that $X$ is a poset. partially ordered by inclusion.

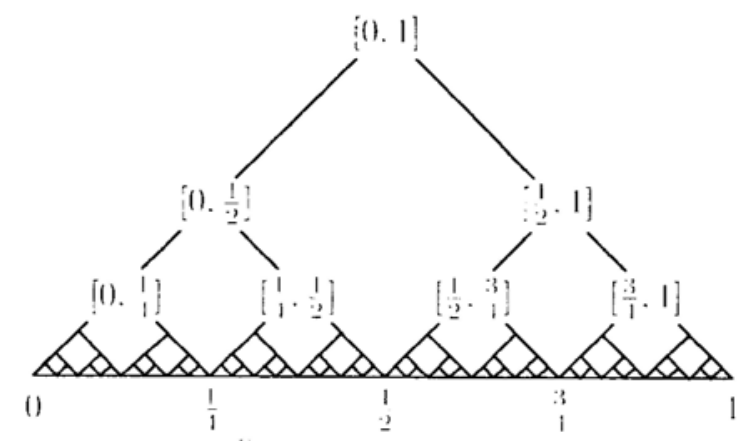

Consider the binary tree formed by starting with the interval $[0,1]$, and successively dividing intervals in half; so an interval $[p, q] \in X$ divides, to form two new intervals $\left[p, \frac{p+q}{2}\right],\left[\frac{p+q}{2}, q\right]$, also in $X$. It is clear that, for any two elements of $X$, either one is contained in the other, or their intersection is either empty or a singleton.

Any infinite strictly descending chain of intervals in $X$ has intersection a singleton subset of $[0,1]$, an element of $X$. 
Conversely, any singleton subset of $[0,1]$ is the intersection of exactly one or two such chains. Any rational $q$ in $(0,1)$ of the form $\frac{i}{2^{n}}$ will occur as the midpoint of some interval in $X$, and, from there on, we have a chain in which $q$ is the right endpoint of each term, and another chain in which $q$ is the left endpoint of each term. All other points of $[0,1]$ occur as the intersection of a unique strictly descending infinite chain of intervals in $X$.

For any nonempty subset $x$ of $[0,1]$, let $x *$ denote the set of all elements of $X$ which contain $x$. Thus, if $x$ has more than one element, then $x *$ is a finite, totally ordered chain $x *=\left\{\left[p_{0}, q_{0}\right],\left[p_{1}, q_{1}\right], \ldots,\left[p_{n}, q_{n}\right]\right\}$, where

$$
[0,1]=\left[p_{0}, q_{0}\right] \supset\left[p_{1}, q_{1}\right] \supset \cdots \supset\left[p_{n}, q_{n}\right] \supseteq x .
$$

Here $x *=\left[p_{n}, q_{n}\right] *$. In general, then, $x *=\bar{x} *$, where $\bar{x}$ is the intersection of all elements of $x *$, so $\bar{x} \in X$.

Let $X_{A}$ be the graph, with vertex set $X$, formed by joining two distinct elements of $X$ whenever they have non-empty intersection.

For each non-empty subset $x$ of $[0,1], x *$ is clearly the vertex set of a complete subgraph of $X_{A}$. Moreover, for any $y \in[0,1]$, we have $\{y\} * \in \mathcal{M}_{0} X_{A}$.

We claim that, conversely, each $Y \in \mathcal{M}_{0} X_{A}$ is of the form $\{y\} *$ for some $y \in[0,1]$. Suppose not. Then we have a $Y \in \mathcal{M}_{0} X_{A}$ such that the intersection of its elements is empty. Since $[0,1]$ is compact, and the elements of $Y$ are closed, we see that the intersection of some finite set of elements $y_{0}, \ldots, y_{n}$ of $Y$, is empty. We may assume that no $y_{i}$ contains another $y_{j}$ as a subset, so the intersection of any two distinct $y_{i}$ is either empty or a singleton. But $Y$ is connected, so each pairwise intersection is a singleton. Since the $y_{i}$ are intervals, any two distinct $y_{i}$ meet in an endpoint. This limits the number of intervals to at most two, and the intersection is then non-empty, a contradiction. This proves the claim.

Thus there is a bijection $[0,1] \rightarrow \mathcal{M}_{0} X_{A}, y \mapsto\{y\} *$.

An element $Y$ of $\mathcal{I}_{0} X_{A}$ is a finite intersection of elements of $\mathcal{M}_{0} X_{A}$, so there is a finite subset $\left\{y_{0}, \ldots, y_{n}\right\}$ of $[0,1]$ such that

$$
Y=\left\{y_{0}\right\} * \cap \cdots \cap\left\{y_{n}\right\} *=\left\{y_{0}, \ldots, y_{n}\right\} *=x *,
$$

where $x=\overline{\left\{y_{0}, \ldots, y_{n}\right\}} \in X$. It follows that we have a poset antiisomorphism $X \rightarrow \mathcal{I}_{0} X_{A}, x \mapsto x *$; that is, both the map and its inverse are inclusion-reversing.

Consider any $Y \in \mathcal{I}_{0} X_{A}$, and let $x$ be the least element of $Y$, so $Y=x *$. Recall that $\mathcal{I}_{0}\left(Y, X_{A}\right)$ is the sub-poset $\left\{Z \in \mathcal{I}_{0} X_{A} \mid Z \supset Y\right\}$ 
of $\mathcal{I}_{0} X_{A}$. Then the above poset anti-isomorphism induces a poset antiisomorphism between the subposet $\{z \in X \mid z \subset x\}$ of $X$, and the poset $\mathcal{I}_{0}\left(Y, X_{A}\right)$. Temporarily, let us denote the former poset $(-\infty, x)$.

If $Y \notin \mathcal{M}_{0} X_{A}$, then its least element, $x$, is an interval which is not a singleton, and the poset $(-\infty, x)$ has exactly two maximal elements, namely the two intervals $x_{1}, x_{2}$ formed by dividing $x$. Moreover, there is exactly one element of $(-\infty, x)$ comparable to both $x_{1}$ and $x_{2}$, namely $x_{1} \cap x_{2}$, the singleton which consists of the midpoint of the interval $x$. It follows that the associated simplicial complex $\mathcal{I}_{*+}\left(Y, X_{A}\right)$ is the union of two cones, and the intersection of the two cones is a single point. Such a complex is easily seen to be contractible. Thus we have proved that, if $Y \notin \mathcal{M}_{0} X_{A}$, then $\operatorname{hd}_{k}\left(Y, X_{A}\right)=-\infty$.

On the other hand, if $Y \in \mathcal{M}_{0} X_{A}$, then $\mathcal{I}_{0}\left(Y, X_{A}\right)$ is empty, so $\mathcal{I}_{*}\left(Y, X_{A}\right)$ consists of a -1 simplex, and here $\operatorname{hd}_{k}\left(Y, X_{A}\right)=0$.

Thus, for any $Y \in \mathcal{I}_{0} X_{A}$,

$$
\operatorname{hd}_{k}\left(Y, X_{A}\right)=\left\{\begin{aligned}
0 & \text { if } Y \in \mathcal{M}_{0} X_{A} \\
-\infty & \text { otherwise }
\end{aligned}\right.
$$

Now let $K$ be a field, set $R(\phi)=K$, and, for each $x \in X$, set

$$
R(x)=\left\{\begin{array}{cl}
K\left[e_{x} \mid e_{x}^{2}=e_{x}\right] & \text { if } x \text { is a singleton } \\
K & \text { otherwise }
\end{array}\right.
$$

Consider the family of $K$-algebras $(R(Y) \mid Y \subseteq X)$, defined as in Example $3.3(i)$. For any $Y \in \mathcal{I}_{0} X_{A}$,

$$
R(Y) \approx\left\{\begin{array}{cl}
K \times K & \text { if } Y \in \mathcal{M}_{0} X_{A} \\
K & \text { otherwise }
\end{array}\right.
$$

so l. gl. $\operatorname{dim} . R(Y)=0$.

Hence $\sup \left\{\operatorname{hd}_{k}\left(Y, X_{A}\right)+\right.$ l. gl. $\left.\operatorname{dim} . R(Y) \mid Y \in \mathcal{I}_{0} X_{A}\right\}=0$.

But $R=R(X)$ is the $K$-algebra coproduct of a family of copies of $K\left[e \mid e^{2}=e\right]$, indexed by the elements of $[0,1]$, so $R$ does not have global dimension 0 . (In fact, $R$ must then have global dimension 1, by [2, Corollary 2.5] or [9, Corollary 7].)

Hence the inequality in the conclusion of Corollary 5.7 is not valid for this example.

Let us apply Corollary 5.7 to graph products of groups. 
5.9. Corollary. Let $X_{A}$ be finite-dimensional, and let $(G(x) \mid x \in X)$ be a family of groups indexed by $X$, and let $G=G\left(X_{A}\right)$ be their graph product.

Then there there is a finite exact sequence of left $K G$-modules

$$
\begin{aligned}
\cdots \rightarrow \underset{Y \in \mathcal{I}_{0}\left(X_{A}\right)}{\oplus} c_{n, Y} K[G / G(Y)] & \rightarrow \ldots \\
\cdots & \rightarrow \underset{Y \in \mathcal{I}_{0}\left(X_{A}\right)}{\oplus} c_{0, Y} K[G / G(Y)] \rightarrow K \rightarrow 0,
\end{aligned}
$$

where $c_{n, Y} K[G / G(Y)]$ denotes a direct sum of $c_{n}\left(Y, X_{A}, k\right)$ copies of $K[G / G(Y)]$.

Hence $\operatorname{cd}_{K} G\left(X_{A}\right) \leq \sup \left\{\operatorname{hd}_{k}\left(Y, X_{A}\right)+\operatorname{cd}_{K} G\left(Y_{A}\right) \mid Y \in \mathcal{I}_{0} X_{A}\right\}$.

Bestvina [5], refining techniques of Davis [8], gives a similar result for finitely generated Coxeter groups, and there is some overlap with the above result in the case of right-angled Coxeter groups.

\section{Normal forms and module-freeness adapted from G. M. Bergman [3, Section 2]}

The results of this section shed some light on the structure of the rings with which we have been dealing.

Here we examine the following special case of Hypothesis 3.1.

6.1. Hypothesis. For each $x \in X$, let $R(x)$ be a $K$-ring given with a $K$-centralizing (right) $K$-basis $B(x) \cup\{1\}$, where $1 \notin B(x)$. For each $(x, y) \in A$, let $B(x, y)=\{a b-b a \mid a \in B(x), b \in B(y)\}$. For each subset $Y$ of $X$, let $R(Y)$ be the quotient of $\coprod_{y \in Y} R(y)$ by the ideal generated by the images of the $B(x, y),(x, y) \in A \cap(Y \times Y)$.

The object of this section is to prove that, for any $Y \subseteq X, R=R(X)$ is free as right $R(Y)$-module on a basis containing 1 ; this is stronger than the conclusion of Proposition 3.4. The form taken by the basis of $R_{R(Y)}$ will show in particular that if, for each $x \in X$, we are given a subset $C(x)$ of $B(x) \cup\{1\}$, such that $1 \in C(x)$, and $C(x)$ is the $K$-basis of a subring $S(x)$ of $R(x)$, then $S(X)$, which is defined in the obvious way, is naturally embedded in $R(X)$.

6.2. Examples. (i) If $K$ is a field contained in the center of each $R(x), x \in X$, then $R(x)$ obviously has a $K$-centralizing $K$-basis containing 1 . Thus the situation considered here includes the case of Construction 1.2 where $K$ is a field. 
(ii) In the situation of Construction 1.3, set $R(x)=K[G(x)]$ and $B(x)=G(x)-\{1\}$. Then $B(x) \cup\{1\}$ is a $K$-centralizing $K$-basis of $R(x)$ and $R(X)=K\left[G\left(X_{A}\right)\right]$.

Further, if, for each $x \in X, C(x)$ is a submonoid of the group $G(x)$, then $C(x)$ is the $K$-basis of a subring $S(x)=K[C(x)]$ of $R(x)$, and $S(X)=K\left[C\left(X_{A}\right)\right]$, where $C\left(X_{A}\right)$ is defined in the obvious way, so the above statement implies that $C\left(X_{A}\right) \subseteq G\left(X_{A}\right)$.

Let $B$ denote the disjoint union of the $B(x)$. Each element $b \in B$ will be said to be "associated to" the index $x \in X$ such that $b \in B(x)$. Let $B^{*}$ denote the free monoid on the set $B$.

Since $R$ is generated by the images of the $R(x)$, it will be spanned as right $K$-module by the products of the images of the elements of $B$ (counting the empty product, 1), i.e. by the natural image of $B^{*}$. We shall call these products monomials, and denote them by the same symbols as the elements of $B^{*}$ of which they are images, though the map $B^{*} \rightarrow R$ is generally not one-to-one.

But we will be careful to distinguish between speaking of two monomials as being "equal in $R$ ", and being "equal", which will mean "equal in $B^{* \prime}$.

Note that if a monomial $b_{1} \cdots b_{n}$ has two succesive terms $b_{i}, b_{i+1}$ both associated with the same index $x \in X$, then, by writing the product $b_{i} b_{i+1} \in R(x)$ as a $K$-linear combination of elements of $B(x) \cup\{1\}$, we can reduce $b_{1} \cdots b_{n}$ in $R$ to a $K$-linear combination of monomials of shorter length. More generally, if $b_{1} \cdots b_{n}$ has two terms $b_{i}$ and $b_{j}(i<j)$ associated with the same index $x \in X$, and if all terms $b_{k}$ occuring between these (i.e., $i<k<j$ ) are associated with indices $y$ such that $(x, y) \in A$, then, in $R$, we can commute $b_{i}$ past these terms until it is adjacent to $b_{j}$, and then reduce our monomial as above to a $K$-linear combination of shorter monomials.

We deduce that $R$ will be spanned as a right $K$-module by those monomials $b_{1} \cdots b_{n}$ with the property that any two terms $b_{i}$ and $b_{j}$ therein, that are associated with the same index $x \in X$, are separated by at least one intermediate term $b_{k}$ associated with an index $y$ such that $(x, y) \notin A$. We shall call such $b_{1} \cdots b_{n}$ "acceptable monomials", and denote the set of acceptable monomials $S \subseteq B^{*}$.

An acceptable monomial can still have adjacent terms $b_{i} b_{i+1}$ associated with indices $x$ and $y$ such that $(x, y) \in A$, and in this case it will be equal in $R$ to the (also acceptable) monomial obtained by transposing these terms. To obtain invariants of acceptable monomials under such transpositions, let us associate to any acceptable monomial $b_{1} \cdots b_{n}$ a partial ordering of its terms, setting 
(10) $b_{i} \prec b_{j}$ if $i<j$ and there exists a sequence

$$
i=m_{1}<\cdots<m_{q}<\cdots<m_{r}=j
$$

such that, writing $\operatorname{ind}(q)$ for the index associated with $b_{m_{q}}$, we have (ind $(q), \operatorname{ind}(q+1)) \notin A$ for all $q<r$.

We are being sloppy in our notation, since a monomial may repeat terms of $B$, so that it is not really the terms $b_{i}$ that are being partially ordered, but, if you will, their subscripts $i$; or, if you prefer, the pairs $\left(i, b_{i}\right)$. In any case, the point is that we obtain from our monomial a finite partially ordered set, with its vertices labeled with certain elements of $B$, possibly with repetitions. This partially ordered set will (by (10) and the definition of acceptable monomial) have the properties that any two vertices labelled with elements of $B$ associated to indices $x, y$ such that $(x, y) \notin A$ must be related under our ordering (one $\preceq$ the other; note that this includes the case $x=y$ ); and when one vertex covers another (is a minimal vertex $\succ$ than it), the associated indices in $X$ must be distinct.

6.3. Lemma. Let $w=b_{1} \cdots b_{n}$ and $w^{\prime}=b_{1}^{\prime} \cdots b_{n}^{\prime}$ be acceptable monomials of the same length. Then the following conditions are equivalent:

(a) $w^{\prime}$ can be obtained from $w$ by a series of transpositions of adjacent terms $b_{i}, b_{i+1}$ associated to indices $x, y$ such that $(x, y) \in A$.

(b) There is an isomorphism between the partially ordered sets associated with these two monomials, which preserves the B-labels on the vertices. Equivalently: there exists a permutation $\pi \in \operatorname{Sym}_{n}$ such that for all $i, b_{i}^{\prime}=b_{\pi(i)}$, and for all $i, j$ if $b_{i}^{\prime} \prec b_{j}^{\prime}$ in $w^{\prime}$ then $b_{\pi(i)} \prec b_{\pi(j)}$ in $w$.

Further, when these conditions hold, the isomorphism of (b) (equivalently, $\pi$ ) is unique.

Proof: $(a) \Rightarrow(b)$ : We easily see that each transposition leaves the isomorphism class of the $B$-labeled partially ordered set unchanged.

$(b) \Rightarrow(a)$ : If $\pi$ is not the identity, there will be some $i$ such that $\pi(i)>\pi(i+1)$. We see that $b_{i}$ and $b_{i+1}$ must be unrelated under $\prec$ (otherwise $\pi$ would not respect the partial ordering), so they must be associated with a pair of indices $(x, y) \in A$. Hence we may transpose them, transforming $w$ to a monomial the order of whose terms is "closer" to that of $w^{\prime}$ (fewer pairs of terms $b_{i}, b_{j}$ occuring in different orders). Iterating this procedure, we see that $w$ will be transformed in a finite number of steps into $w^{\prime}$. 
To see the last assertion of the lemma, note that in our partially ordered sets, any two vertices bearing the same label in $B$ must be related under $\prec$. Since the sets are finite, there cannot therefore be more than one order-preserving and label-preserving bijection.

Let us write $w \sim w^{\prime}$ if the equivalent conditions of the above lemma hold. This gives an equivalence relation on the set $S$ of acceptable monomials. We shall write $S / \sim=S^{\prime}$, and represent the equivalence class of $w$ by $[w] \in S^{\prime}$. Clearly, the map $S \rightarrow R$ factors through $S^{\prime}$. We shall soon see that this map is one-to-one, and its image is a $K$-centralizing $K$-basis of $R$. But first we need a result on the structure of $S^{\prime}$.

For any subset $Y$ of $X$, let us define $S_{Y}^{\prime}$ to be the set of all $\left[b_{1} \cdots b_{n}\right] \in S^{\prime}$ such that all $b_{i} \in \underset{x \in Y}{\cup} B(x)$. Let us also define $S_{\urcorner Y}^{\prime}$ to be the set of all $[w] \in S^{\prime}$ such that in the partially ordered set associated with $[w]$, no maximal vertex is labeled with a member of any $B(y)$ with $y \in Y$. We note that the maximal vertices of the partially ordered set associated with $[w]$ correspond to those terms that can be transposed to the rightmost position. (E.g., if $(x, y),(x, z) \in A, y \neq z, a \in B(x)$, $b \in B(y), c \in B(z)$, then, in the partially ordered set associated with [abc], $a$ and $c$ are both maximal.)

Note that if $\left[w_{1}\right] \in S_{\urcorner Y}^{\prime}$ and $\left[w_{2}\right] \in S_{Y}^{\prime}$ then $w_{1} w_{2}$ will be an acceptable monomial. Further, $\left[w_{1} w_{2}\right]$ will be determined by the equivalence classes $\left[w_{1}\right]$ and $\left[w_{2}\right]$, since any transposition of terms that can be performed in the latter elements can certainly be duplicated in the product. In fact, we have:

6.4. Lemma. Let $Y \subseteq X$. Then for any element $[w] \in S^{\prime}$ there exist unique elements $\left[w_{1}\right] \in S_{\urcorner_{Y}^{\prime}}^{\prime},\left[w_{2}\right] \in S_{Y}^{\prime}$ such that $[w]=\left[w_{1} w_{2}\right]$.

Proof: To get the existence of such a decomposition, look for a maximal term of $w$ associated with an index in $Y$; if there is one, transpose it to the last position. Then treat the remaining string of terms (shorter by one) the same way. It may contain maximal terms that were not maxi$\mathrm{mal}$ in the original element, because they were covered by the first term extracted. Iterate until we are left with a string $w_{1}$ (possibly empty) with no maximal terms associated with an index in $Y$, followed by a string $w_{2}$ with all terms associated to indices in $Y$.

To get uniqueness, note that $w_{2}$ must consist precisely of those terms of $w$ which are associated to indices in $Y$, and are not $\prec$ any terms with indices in $X-Y$.

We can now prove: 
6.5. Proposition. If Hypothesis 6.1 holds, then the images in $R$ of the distinct elements of $S^{\prime}$ are distinct, and form a K-centralizing $K$-basis of $R$.

Proof: Let $M$ be a free right $K$-module on the basis $S^{\prime}$. We shall show that $M$ may be made a right $R$-module in a natural way, and that the actions on this module of distinct elements of $S^{\prime}$ are right $K$-linearly independent. (The idea of this trick goes back to van der Waerden, cf. $\left[4\right.$, Section 11.2, $\left.\left.\left(28^{\prime \prime}\right)\right]\right)$.

For each $x \in X$, take $Y=\{x\}$ in Lemma 6.4. Every member of $S_{\{x\}}^{\prime}$ is of the form $[b](b \in B(x))$, or [1], so the lemma says that we get a bijection $S_{\urcorner\{x\}}^{\prime} \times(B(x) \cup\{1\}) \rightarrow S^{\prime}$, given by $\left(\left[w_{1}\right], b\right) \mapsto\left[w_{1} b\right]$. But $B(x) \cup\{1\}$ is a right $K$-basis for $R(x)$, so this decomposition allows us to give the free right $K$-module $M$ on $S^{\prime}$ a structure of free right $R(x)$-module on the basis $S_{\neg\{x\}}^{\prime}$, extending the given right $K$-module structure. Doing this for all $x \in X$, we get a structure of right $\coprod_{x \in X} R(x)$-module.

Now take any $(x, y) \in A$. We see that $S_{\{x, y\}}^{\prime}$ will consist of elements $\left[w_{x} w_{y}\right]=\left[w_{y} w_{x}\right], w_{x} \in B(x) \cup\{1\}, w_{y} \in B(y) \cup\{1\}$. (Because elements of $B(x)$ and $B(y)$ are transposable with one another, we can form from them no acceptable monomial of length greater than two, by the definition of acceptable monomial.) This gives a bijection

$$
S_{\urcorner\{x, y\}}^{\prime} \times(B(x) \cup\{1\}) \times(B(y) \cup\{1\}) \rightarrow S^{\prime} .
$$

Since $(B(x) \cup\{1\}) \times(B(y) \cup\{1\})$ commutes with $K$, we see that $S_{\urcorner\{x, y\}}^{\prime}$ is a right $K$-basis of $R(\{x, y\})$. Thus we may define a structure of (free) right $R(\{x, y\})$-module on $M$ which, clearly, extends the structures of right $R(x)$ - and $R(y)$-module already defined. This means that the actions of $B(x)$ and the actions of $B(y)$ on $M$ must commute with one another. Since this is so for all pairs $(x, y) \in A$, our right $\coprod_{x \in X} R(x)$-module structure must in fact give a right $R(X)$-module structure, by the definition of $R(X)$.

Now note that for any $\left[b_{1} \cdots b_{n}\right] \in S^{\prime}$, the product $b_{1} \cdots b_{n} \in R$, acting on the element $[1] \in M$, will give $\left[b_{1} \cdots b_{n}\right] \in M$. It follows that any non-trivial $K$-linear combination in $R$ of the images of elements of $S^{\prime}$ are $K$-linearly independent. Since we already know they span $R$, this completes the proof of the proposition.

We can now get our desired freeness result from Lemma 6.4.

6.6. Proposition. If Hypothesis 6.1 holds then, for any subset $Y$ of $X, R(X)$ is free as a right module over $R(Y)$, with basis the (faithful) image of $S_{\urcorner Y}^{\prime}$. 
Acknowledgements. We thank George Bergman for the ideas and extensive contributions which were fundamental to this work, and for permission to include section 6 , which closely follows [3, Section 2].

We also thank Michael Barr for suggesting, in 1976, that an example based on the projective plane should yield 2-torsion; we took a 16 vertex, full, triangulation of the projective plane, took the dual polygonal tessellation, completed each $n$-gon to an $(n-1)$-simplex, and took the 1 -skeleton, a graph with 32 vertices. The $\phi$-component of the corresponding refined resolution was worked out by the Bedford College Computer Centre between March 1977 and June 1978; it was found to be the desired multiplication-by-2 map, as in Example 2.5. We are grateful to the programmers Tom Lake and Phil Taylor for their intense efforts. Bestvina's recent, elegant, approach [5, Remark (3)], shows that one can simply take the 1-skeleton of a full triangulation; this led us to Example 2.5, which does not require a computer. We thank P. H. Kropholler for illuminating conversations concerning Bestvina's article.

The authors gratefully acknowledge that this research was generously funded by the DGICYT, through grant PB90-0719 for the first-named author, and a post-doctoral fellowship held at the Centre de Recerca Matemàtica for the second-named author.

\section{References}

1. Y.-G. BAIK, J. HowIE, S. J. PrIDE, The identity problem for graph products of groups, J. Algebra 162 (1993), 168-177.

2. G. M. Bergman, Modules over coproducts of rings, Trans. Amer. Math. Soc. 200 (1974), 1-32.

3. G. M. Bergman, The global dimension of mixed coproduct/tensor product algebras, (Unpublished note, 1976, 19 pages).

4. G. M. Bergman, The diamond lemma for ring theory, Advances in Mathematics 29 (1978), 178-218.

5. M. Bestvina, The virtual cohomological dimension of Coxeter groups, London Math. Soc. Lecture Notes 181 (1993), 19-23.

6. I. M. ChISwell, The Euler characteristic of graph products and of Coxeter groups, London Math. Soc. Lecture Notes 173 (1992), 36-46.

7. P. M. CoHN, On the free product of associative rings, Math. Zeits. 71 (1959), 380-398.

8. M. W. Davis, Groups generated by reflections, Ann. of Math. 117 (1983), 293-324. 
9. W. Dicks, Mayer-Vietoris presentations over colimits of rings, Proc. London Math. Soc.(3) 34 (1977), 557-586.

10. W. Dicks, An exact sequence for rings of polynomials in partly commuting indeterminates, J. Pure and Applied Algebra 22 (1981), 215-228.

11. E. R. GReEN, "Graph products of groups," Ph. D. Thesis, University of Leeds, 1990.

12. A. WeIl, Sur les théorèmes de de Rham, Comment. Math. Helv. 26 (1952), 119-145.

\author{
Warren Dicks: \\ Departament de Matemàtiques \\ Universitat Autònoma de Barcelona \\ 08193 Bellaterra (Barcelona) \\ SPAIN
}

\author{
I. J. Leary: \\ Centre de Recerca Matemàtica \\ Apartat 50 \\ 08193 Bellaterra (Barcelona) \\ SPAIN
}

Primera versió rebuda el 7 de Juliol de 1993, darrera versió rebuda el 10 de Gener de 1994 